\title{
Modeling Transient Long Waves Propagating through a Heterogeneous Coastal Forest of Arbitrary Shape
}

\author{
Che-Wei Chang ${ }^{1, *}$, Philip L.-F. Liu ${ }^{1,2,3}$, Chiang C. Mei ${ }^{4}$, Maria Maza ${ }^{5}$ \\ ${ }^{1}$ School of Civil and Environmental Engineering, Cornell University, USA \\ 2 Department of Civil and Environmental Engineering, National University of Singapore, Singapore \\ ${ }^{3}$ Institute of Oceanic and Hydrological Sciences, National Central University, Taiwan \\ ${ }^{4}$ Department of Civil and Environmental Engineering, MIT, USA \\ ${ }^{5}$ Instituto de Hidraulica Ambiental, Universidad de Cantabria, Spain \\ * Corresponding Author (Email: cc2338@cornell.edu)
}

\begin{abstract}
A model is proposed to study transient long waves propagating through coastal vegetation. The coastal forest is modeled by an array of rigid and vertically surface-piercing cylinders. The homogenization method, i.e. multi-scale perturbation theory, is applied to separate two contrasting physical length scales: the scale characterizing transient waves and the scale representing the diameter of and the spacing among cylinders. Fourier transform is employed so that all the free surface elevation and velocity field are solved in the frequency domain. For each harmonic, the flow motion within a unit cell, consisting of one or more cylinders, is obtained by solving the micro-scale boundary-value problem, which is driven by the macro-scale (wavelength scale) pressure gradients. The cell-averaged equations governing the macro-scale wave amplitude spectrum are derived with the consideration of the effects of the cell problem solution. Similar to Chang, Liu, Mei, and Maza (2016), the macro-scale wave amplitude spectrum is solved numerically with the boundary integral equation method, where a vegetated area is composed of multiple patches of arbitrary shape. Each forest patch can be divided into subzones according to different properties, such as planting pattern and vegetation size. Each subzone is considered as a homogeneous forest region with a constant bulk eddy viscosity determined by the empirical formula suggested in Mei, Chan, Liu, Huang, and Zhang (2011). Once the solutions for wave amplitude spectrum are obtained, the free surface elevation can then be computed from the inverse Fourier transform. A computing program is developed based on the present numerical model. To check the present approach, we investigate several different forest configurations. However, we focus on incident waves with a solitonlike shape. We first re-examine the forest belt case. The numerical model is then checked by available theoretical results along with experimental measurements for two special forest configurations. For a single circular forest, the numerical results compare almost perfectly with the analytical solutions. The comparison with experimental data also shows very good agreements. The effects of different wave parameters on damping rate are discussed. The numerical model is further compared with the experiments for a forest region consisting of multiple circular patches. Good agreements are also observed between the simulated free surface elevations and the experimental measurements. The effectiveness of these two forest configurations on wave attenuation is discussed.
\end{abstract}

\section{Introduction}

Coastal vegetation has been considered as an effective means of shore protection for both short waves and long waves. To estimate wave attenuation by vegetation, laboratory observations as well as mathematical modeling on waves through coastal forests have been widely studied in recent years. Most of the existing literatures have been reviewed in Mei et al. (2011, 2014). However, in the interest of understanding the capability of coastal vegetation (e.g., mangroves) to dissipate long waves (e.g., tsunami waves, storm surges),

(C) 2016. This manuscript version is made available under the Elsevier user license http://www.elsevier.com/open-access/userlicense/1.0/ 
several recent studies are worthy of mentioning here. For example, Huang et al. (2011) conducted laboratory experiments on solitary waves through an array of emergent rigid cylinders. Different cylinder arrangements and sizes of array were tested. By measuring the free surface elevation, the solitary wave evolution across the target cylinder array was reported and the wave attenuation was shown. In addition, Irtem et al. (2009)'s experiments, using both artificial trees and cylindrical timber sticks, demonstrated the effectiveness of a coastal forest on reducing the maximum run-up of tsunami waves. Ismail et al. (2012) also examined the influences of vegetation density on wave dissipation and tsunami run-up in their experiments. Different parameterized models of mangroves were adopted in tsunami damping tests as well (e.g., Husrin et al. 2012 and Strusinska-Correia et al. 2013). Numerical approaches on interactions between solitary waves and vegetation have also been developed. For example, Maza et al. (2015) proposed a three dimensional numerical scheme, directly simulating the interaction of solitary waves with emergent rigid cylinders. The flow field around each cylinder was computed from the direct simulation; however, the required computational efforts were considerable.

As discussed in Liu et al. (2015), applying homogenization (multi-scale perturbation) theory provides higher computational efficiency compared with direct numerical approach. Separating the wave motion into two sharply contrasting scales, the macro-scale wave dynamics is solved based on cell-averaged equations, allowing better understanding of the physical process. For periodic waves, the semi-analytical model has been established for predicting the wave attenuation by specific configurations of cylinder array, e.g. an infinitely long forest belt (Mei et al. 2011, 2014) and a circular forest (Liu et al. 2015). Furthermore, Chang et al. (2016) extended the model to a forest region of arbitrary shape by employing the boundary integral equation method. For transient waves, on the other hand, the model developed in Mei et al. (2011) is limited to incident soliton-like waves propagating through a forest belt.

In this paper, we follow Chang et al. (2016) and apply the homogenization theory to a transient long wave propagating through a forest region, which is composed of multiple patches of arbitrary shape. Each forest patch has different numbers of subzones due to different properties (e.g. vegetation type/size, arrangement and porosity). A subzone can be surrounded by other subzones and/or the open water region, where a sketch of a general forest region has been given in Chang et al. (2016)'s Fig.1 (it is also shown as Fig.S1 in the Supplementary Materials). With the assumption of homogeneous forest subzones, a dimensional bulk eddy viscosity is obtained by modifying the empirical formula in Mei et al. (2011). Fourier transform along with the boundary integral equation methods are applied such that the arbitrary shape of patches/subzones can be resolved and the macro-scale wave amplitude spectrum is solved numerically. The free surface elevation is then obtained by inversely transforming the wave amplitude spectrum solutions back into the physical domain.

To check the present approach, several forest configurations have been investigated. For simplicity only the incident waves of soliton-like shape are considered. The forest belt studied in Mei et al. (2011) is first re-examined with new analytical solutions. A homogeneous circular forest, used in Liu et al. (2015) and Chang et al. (2016) for studying periodic waves, is then employed to check the present approach and numerical model. Negligible differences are observed between the analytical solutions and the numerical results for these cases. The comparison between model results and experimental data is also presented, showing very good agreement. The relationship between the damping rate and the nonlinearity of incident wave is discussed. For further investigation of the skill of the numerical model, the experiments on multiple circular forest patches in Maza (2015) and Maza et al. (2016) are simulated and good agreements with the experimentally-observed free surface elevations are obtained. The comparison of the effectiveness of a circular forest and multiple smaller patches on damping the incident wave is presented.

\section{Formulation}

Defining $\vec{x}=\left(x_{1}, x_{2}\right)$ as the horizontal Cartesian coordinates and $z$ as the vertical coordinate pointing upwards from the mean water level, the free surface elevation can be expressed as $z=\eta(\vec{x}, t)$. Focusing on small-amplitude long waves, propagating in a constant water depth $h_{0}$, the leading-order velocities are on the horizontal plane and are independent of $z$, i.e. $u_{i}(\vec{x}, t), i=1,2$. Therefore, the linearized equations (Mei et al. 2011) can be written as

$$
\frac{\partial \eta}{\partial t}+h_{0} \frac{\partial u_{i}}{\partial x_{i}}=0
$$


and

$$
\frac{\partial u_{i}}{\partial t}=-g \frac{\partial \eta}{\partial x_{i}}+\nu_{e}\left(\frac{\partial^{2} u_{i}}{\partial x_{j} \partial x_{j}}\right)
$$

in which the bottom friction has been neglected and the eddy viscosity $\nu_{e}$ is assumed as a constant.

In this paper we shall use the Fourier transform method to solve the governing equations and boundary conditions. As an example, the Fourier transform of the free surface elevation $\eta$ can be expressed as

$$
\hat{\eta}\left(x_{i}, \omega\right)=\int_{-\infty}^{\infty} \eta\left(x_{i}, t\right) e^{\mathrm{i} \omega t} d t
$$

where $\hat{\eta}\left(x_{i}, \omega\right)$ is called wave amplitude spectrum. The inverse Fourier transform of the wave amplitude spectrum gives back the expression of the wave surface profile:

$$
\eta\left(x_{i}, t\right)=\frac{1}{2 \pi} \int_{-\infty}^{\infty} \hat{\eta}\left(x_{i}, \omega\right) e^{-\mathrm{i} \omega t} d \omega .
$$

Here we introduce the dimensionless variables as

$$
x_{i}^{*}=\frac{x_{i}}{\ell}, \quad t^{*}=\omega t, \quad \eta^{*}=\frac{\eta}{H_{\mathrm{inc}}} \quad \text { and } \quad u_{i}^{*}=\frac{u_{i}}{\sqrt{g\left(h_{0}+H_{\mathrm{inc}}\right)} H_{\mathrm{inc}} /\left(h_{0}+H_{\mathrm{inc}}\right)}
$$

where the horizontal coordinates are scaled by the characteristic tree spacing $\ell$ (micro-length) and the free surface elevation is scaled by the incident wave height $H_{\text {inc }}$. The wave speed of a solitary wave, i.e. $\sqrt{g\left(h_{0}+H_{\text {inc }}\right)}$, is assumed. We first apply Fourier transform to the linearized equations (2.1) and (2.2), and then normalize the results with the proper scales as indicated in (2.5) to yield

$$
-\mathrm{i} \varepsilon \hat{\eta}^{*}+\frac{\partial \hat{u}_{i}^{*}}{\partial x_{i}^{*}}=0, \quad i, j=1,2
$$

and

$$
-\mathrm{i} \varepsilon \hat{u}_{i}^{*}=-\frac{\partial \hat{\eta}^{*}}{\partial x_{i}^{*}}+\varepsilon \sigma \frac{\partial^{2} \hat{u}_{i}^{*}}{\partial x_{j}^{*} \partial x_{j}^{*}}, \quad i, j=1,2
$$

where $\hat{u}_{i}^{*}$ is the dimensionless velocity spectrum and the small paramter $\varepsilon$ is defined as

$$
\varepsilon=\frac{\omega \ell}{\sqrt{g\left(h_{0}+H_{\text {inc }}\right)}} \equiv k \ell \ll \mathcal{O}(1) .
$$

We remark here that since the linear long wave theory is used, the incident wave height must be reasonably small, i.e. $H_{\text {inc }} / h_{0} \ll \mathcal{O}(1)$ has been applied. The wave dispersion is also assumed to be negligible. Therefore, the size of the forest should be in the same order of magnitude of the characteristic wavelength, which has to be much greater than the tree spacing.

Following Mei et al. (2011), we propose that each forest subzone has a constant bulk value of dimensional eddy viscosity $\nu_{e}$, which can be determined by the following empirical formula:

$$
\nu_{e}=1.86(1-n)^{2.06} U_{0} \ell \quad \text { with } \quad U_{0}=\sqrt{g\left(h_{0}+H_{\mathrm{inc}}\right)}\left(\frac{H_{\mathrm{inc}}}{h_{0}+H_{\mathrm{inc}}}\right)
$$

where $U_{0}$ represents the depth-averaged horizontal water particle velocity. The corresponding dimensionless eddy viscosity can then be written as

$$
\sigma=\frac{\nu_{e}}{\omega \ell^{2}}=1.86(1-n)^{2.06} \frac{1}{k \ell}\left(\frac{H_{\mathrm{inc}}}{h_{0}+H_{\mathrm{inc}}}\right), \quad k=\frac{\omega}{\sqrt{g\left(h_{0}+H_{\mathrm{inc}}\right)}} .
$$

The dimensionless eddy viscosity $\sigma$ is a function of $\omega$. It should be noted that a similar formula was used in Mei et al. (2011), where $U_{0}=\sqrt{g h_{0}} H_{\text {inc }} / 2 h_{0}$ was specified.

In the paper we shall focus on the soliton-like incident waves. The free surface profile can be expressed as

$$
\eta_{\text {inc }}\left(x_{1}, t\right)=H_{\mathrm{inc}} \operatorname{sech}^{2}\left[k_{s}\left(x_{1}-c t\right)\right]
$$


where the effective wavenumber $k_{s}$ and the wave speed $c$ are defined as

$$
k_{s}=\frac{1}{h_{0}} \sqrt{\frac{3 H_{\mathrm{inc}}}{4 h_{0}}} \text { and } c=\sqrt{g\left(h_{0}+H_{\mathrm{inc}}\right)} .
$$

The incident soliton has the wave amplitude spectrum (Miles 1976) as

$$
\hat{\eta}_{\text {inc }}\left(x_{1}, \omega\right)=A_{s} e^{\mathrm{i} k x_{1}}, \quad A_{s}(\omega)=\frac{4}{3} \pi h_{0}^{3}\left(\frac{\omega}{c^{2}}\right) \operatorname{csch}\left[\pi\left(\frac{h_{0}^{3}}{3 H_{\text {inc }}}\right)^{1 / 2} \frac{\omega}{c}\right]
$$

in which $k=\omega / c$. For an incident soliton, where the wave amplitude spectrum is an even function in $\omega$, the inverse Fourier transform in (2.4) can also be modified as

$$
\eta\left(x_{i}, t\right)=\frac{1}{\pi} \int_{0}^{\infty} \hat{\eta}\left(x_{i}, \omega\right) e^{-\mathrm{i} \omega t} d \omega .
$$

Note that in the numerical computations, the infinite components of harmonic $\omega$ needs to be truncated to a finite number with proper convergent test. The details will be discussed later in Sec.3.4.

Here we introduce two coordinates to capture the physics of the flows in the vicinity of cylinders and the wave motions propagating through the entire forest region:

$$
X_{i}^{*}=\varepsilon x_{i}^{*}=k x_{i} .
$$

Employing the multiple-scale perturbation method and dropping the asterisks for brevity, the dimensionless velocity and wave amplitude spectrum can be expressed as

$$
\hat{u}_{i}=\hat{u}_{i}^{(0)}+\varepsilon \hat{u}_{i}^{(1)}+\varepsilon^{2} \hat{u}_{i}^{(2)}+\cdots \quad \text { and } \quad \hat{\eta}=\hat{\eta}^{(0)}+\varepsilon \hat{\eta}^{(1)}+\varepsilon^{2} \hat{\eta}^{(2)}+\cdots
$$

in which $\hat{u}_{i}^{(n)}$ and $\hat{\eta}^{(n)}$, with $n=0,1,2, \cdots$, are functions of $\left(x_{i}, X_{i}, \omega\right)$. Note again that only dimensionless variables are presented from here on.

\subsection{Micro-scale (cell) Problem}

To solve the flow motions around cylinders within a unit cell, we express the leading-order velocities and the free surface fluctuation in terms of the convolution integrals (Mei and Vernescu 2010; Mei et al. 2011) as:

$$
u_{i}^{(0)}(\vec{x}, \vec{X}, t)=-\int_{0}^{t} K_{i j}(\vec{x}, t-\tau) \frac{\partial \eta^{(0)}(\vec{X}, \tau)}{\partial X_{j}} d \tau, \quad \vec{x} \in \Omega_{\mathrm{f}}
$$

and

$$
\eta^{(1)}(\vec{x}, \vec{X}, t)=-\int_{0}^{t} A_{j}(\vec{x}, t-\tau) \frac{\partial \eta^{(0)}(\vec{X}, \tau)}{\partial X_{j}} d \tau, \quad \vec{x} \in \Omega_{\mathrm{f}}
$$

For transient waves, $K_{i j}, A_{j}$ and $\eta^{(0)}$ are nonzero when time is greater than or equal to zero. (2.17) and (2.18) can be rewritten in the general convolution form by integrating from negative infinity to positive infinity:

$$
u_{i}^{(0)}(\vec{x}, \vec{X}, t)=-\int_{-\infty}^{\infty} K_{i j}(\vec{x}, t-\tau) \frac{\partial \eta^{(0)}(\vec{X}, \tau)}{\partial X_{j}} d \tau, \quad \vec{x} \in \Omega_{\mathrm{f}}
$$

and

$$
\eta^{(1)}(\vec{x}, \vec{X}, t)=-\int_{-\infty}^{\infty} A_{j}(\vec{x}, t-\tau) \frac{\partial \eta^{(0)}(\vec{X}, \tau)}{\partial X_{j}} d \tau, \quad \vec{x} \in \Omega_{\mathrm{f}}
$$

Applying Fourier transform to (2.19) and (2.20), the convolution property provides the transformed relationships as

$$
\hat{u}_{i}^{(0)}=-\hat{K}_{i j} \frac{\partial \hat{\eta}^{(0)}}{\partial X_{j}} \quad \text { and } \quad \hat{\eta}^{(1)}=-\hat{A}_{j} \frac{\partial \hat{\eta}^{(0)}}{\partial X_{j}}, \quad \vec{x} \in \Omega_{\mathrm{f}}
$$


where $\Omega_{\mathrm{f}}$ represents the fluid part within a unit cell $\Omega$. From the mass conservation of $\mathcal{O}(1)$ and the momentum equations of $\mathcal{O}(\varepsilon), \hat{K}_{i j}$ and $\hat{A}_{j}$ satisfy

$$
\frac{\partial \hat{K}_{i j}}{\partial x_{i}}=0
$$

and

$$
\mathrm{i} \hat{K}_{i j}=-\delta_{i j}+\frac{\partial \hat{A}_{j}}{\partial x_{i}}-\sigma \frac{\partial^{2} \hat{K}_{i j}}{\partial x_{k} \partial x_{k}}, \quad \vec{x} \in \Omega_{\mathrm{f}}
$$

in which $\hat{K}_{i j}$ and $\hat{A}_{j}$ are subject to the cell-periodicity (Mei et al. 2011). $\delta_{i j}$ denotes the Kronecker delta. The no-slip boundary conditions along the solid surface of cylinders are also valid. As noted, in this paper we consider each forest subzone as a homogeneous area where a dimensional bulk eddy viscosity $\nu_{e}$ is determined by (2.9), while the corresponding dimensionless eddy viscosity $\sigma$ in (2.10) vary with different harmonics. Accordingly, within a forest subzone the cell problem for each harmonic has to be solved separately. More details of the numerical solutions to the cell problem can be found in Liu et al. (2015) and Mei et al. (2014).

\subsection{Macro-scale (wavelength-scale) Problem}

With the cell problem solution, the leading-order cell-averaged velocity spectrum can be obtained from $(2.21)$ :

$$
\left\langle\hat{u}_{i}^{(0)}\right\rangle=-\left\langle\hat{K}_{i j}\right\rangle \frac{\partial \hat{\eta}^{(0)}}{\partial X_{j}}
$$

and the cell-averaged mass equation of $\mathcal{O}(\varepsilon)$ gives

$$
-\mathrm{i} n \hat{\eta}^{(0)}+\frac{\partial\left\langle\hat{u}_{i}^{(0)}\right\rangle}{\partial X_{i}}=0
$$

in which Gauss's theorem and the cell-periodic conditions are invoked with the cell porosity $n$ being defined as

$$
n=\frac{\Omega_{\mathrm{f}}}{\Omega}
$$

The above cell-averaged quantity $\langle\cdot\rangle$ is defined by

$$
\langle f\rangle=\frac{1}{\Omega} \iint_{\Omega_{\mathrm{f}}} f d x_{1} d x_{2} .
$$

Combining (2.24) and (2.25), the leading-order cell-averaged equation governing the wave amplitude spectrum becomes

$$
-\mathrm{i} n \hat{\eta}^{(0)}-\hat{\mathcal{K}}\left(\frac{\partial^{2} \hat{\eta}^{(0)}}{\partial X_{i} \partial X_{i}}\right)=0
$$

in which $\left\langle\hat{K}_{i j}\right\rangle=\hat{\mathcal{K}} \delta_{i j}$ has been applied. In the absence of forests, the porosity becomes unity (i.e. $n=1$ ) and $\hat{K}_{i j} \rightarrow \mathrm{i} \delta_{i j}$ following from (2.23). Therefore, in the open water region the governing equation becomes

$$
\hat{\eta}^{(0)}+\frac{\partial^{2} \hat{\eta}^{(0)}}{\partial X_{i} \partial X_{i}}=0
$$

\section{Boundary Integral Equation Method}

Following Chang et al. (2016), we employ the boundary integral equation method to solve the macro-scale wave amplitude spectrum. The integral formulations for a forest subzone as well as for the open water region are both presented. The required matching conditions along the interfaces of two adjacent subzones and the boundary between forest and open water are introduced. 


\subsection{Integral formulation for a forest subzone}

Let us first consider a forest subzone $\xi$ of an arbitrary shape. As sketched in Fig.1c of Chang et al. (2016) (also shown as Fig.S1c in the Supplementary Materials), a forest subzone can be adjacent to other subzones and/or the open water. The equation governing the macro-scale wave amplitude spectrum, as shown in (2.28), is rewritten as

$$
\frac{\partial^{2} \hat{\eta}_{\xi}^{(0)}}{\partial X_{1} \partial X_{1}}+\frac{\partial^{2} \hat{\eta}_{\xi}^{(0)}}{\partial X_{2} \partial X_{2}}+\hat{\beta}^{2} \hat{\eta}_{\xi}^{(0)}=0, \quad \hat{\beta}=\sqrt{\frac{i n}{\hat{\mathcal{K}}}}
$$

Comparing (3.1) with (3.4) in Chang et al. (2016), they are both well-known Helmholtz equations but with different complex coefficients in front of the 3rd term of the equation, which contain information from the respective cell problem solutions. In (3.1) the wave amplitude spectrum $\hat{\eta}_{\xi}^{(0)}$ depends on the frequency $(\omega)$ and will be solved independently for each $\omega$. We convert (3.1) into the integral equation (Liggett and Liu 1983) as

$$
\hat{\eta}_{\xi}^{(0)}\left(\vec{X}_{i}\right)=\frac{-\mathrm{i} \pi}{2 \alpha} \int_{\mathrm{S}_{\xi}+\mathrm{D}_{\xi \psi}}\left\{\hat{\eta}_{\xi}^{(0)}\left(\vec{X}_{j}\right) \frac{\partial\left[H_{0}^{(1)}(\hat{\beta} \underline{r})\right]}{\partial \hat{n}_{\xi}}-H_{0}^{(1)}(\hat{\beta} \underline{r}) \frac{\partial \hat{\eta}_{\xi}^{(0)}\left(\vec{X}_{j}\right)}{\partial \hat{n}_{\xi}}\right\} d S
$$

where the boundary of subzone $\xi$ can include the boundary to open water (i.e. $\mathrm{S}_{\xi}$ ) and/or the interfaces with other subzones $\psi$ (i.e. $\mathrm{D}_{\xi \psi}$ ). $\vec{X}_{i}$ denotes the coordinate of a point on the boundary of the subzone with its interior angle $\alpha$ sketched in Chang et al. (2016)'s Fig.2 (also shown as Fig.S2 in the Supplementary Materials). $\vec{X}_{j}$ is another boundary point of the subzone with $r=\left|\vec{X}_{i}-\vec{X}_{j}\right|$ yielding the distance between these two boundary points. $H_{0}^{(1)}$ is the Hankel function of the first kind and $\hat{n}_{\xi}$ represents the outward normal direction to the boundary of subzone $\xi$. Note again that $\hat{\beta}$ is obtained from the cell problem solution and (3.2) will be used to solve the wave amplitude spectrum as well as its normal derivative along the boundary. After obtaining the wave amplitude spectrum and its normal derivative along the boundary, the solutions inside the subzone can be readily computed by assigning $\vec{X}_{i}$ as an interior point with its interior angle $=2 \pi$ :

$$
\hat{\eta}_{\xi}^{(0)}\left(\vec{X}_{i}\right)=\frac{-\mathrm{i}}{4} \int_{\mathrm{S}_{\xi}+\mathrm{D}_{\xi \psi}}\left\{\hat{\eta}_{\xi}^{(0)}\left(\vec{X}_{j}\right) \frac{\partial\left[H_{0}^{(1)}(\hat{\beta} \underline{r})\right]}{\partial \hat{n}_{\xi}}-H_{0}^{(1)}(\hat{\beta} \underline{r}) \frac{\partial \hat{\eta}_{\xi}^{(0)}\left(\vec{X}_{j}\right)}{\partial \hat{n}_{\xi}}\right\} d S .
$$

Note that $\vec{X}_{i}$ is now an interior point of subzone $\xi$ while $\vec{X}_{j}$ is on its boundary.

\subsection{Integral formulation for open water}

For open water the governing equation is given in (2.29) with subscript $\mathrm{S}$ denoting the scattered wave amplitude spectrum:

$$
\frac{\partial^{2} \hat{\eta}_{\mathrm{S}}^{(0)}}{\partial X_{1} \partial X_{1}}+\frac{\partial^{2} \hat{\eta}_{\mathrm{S}}^{(0)}}{\partial X_{2} \partial X_{2}}+\hat{\eta}_{\mathrm{S}}^{(0)}=0
$$

Equation (3.4) has the same form as (3.10) in Chang et al. (2016) with their coefficient of the 3rd term, $k_{p}$, being replaced by $1 . \hat{\eta}_{\mathrm{S}}^{(0)}$ in (3.4) for each harmonic is solved separately. Equation (3.4) can also be converted into the following integral equation:

$$
\hat{\eta}_{\mathrm{S}}^{(0)}\left(\vec{X}_{i}\right)=\frac{-\mathrm{i} \pi}{2(2 \pi-\alpha)} \int_{\mathrm{S}}\left\{\hat{\eta}_{\mathrm{S}}^{(0)}\left(\vec{X}_{j}\right) \frac{\partial\left[H_{0}^{(1)}(\underline{r})\right]}{\partial \hat{n}_{\mathrm{S}}}-H_{0}^{(1)}(\underline{r}) \frac{\partial \hat{\eta}_{\mathrm{S}}^{(0)}\left(\vec{X}_{j}\right)}{\partial \hat{n}_{\mathrm{S}}}\right\} d S
$$

where $\vec{X}_{i}$ and $\vec{X}_{j}$ are both located on the boundary between open water and forest region. $\mathrm{S}$ denotes the combination of the external boundaries of all the forest patches. Again, once the wave amplitude spectrum $\hat{\eta}_{\mathrm{S}}^{(0)}$ and its normal derivates along the boundary $\mathrm{S}$ are solved, we can assign $\vec{X}_{i}$ as any interior point within the open water region and modify (3.5) as

$$
\hat{\eta}_{\mathrm{S}}^{(0)}\left(\vec{X}_{i}\right)=\frac{-\mathrm{i}}{4} \int_{\mathrm{S}}\left\{\hat{\eta}_{\mathrm{S}}^{(0)}\left(\vec{X}_{j}\right) \frac{\partial\left[H_{0}^{(1)}(\underline{r})\right]}{\partial \hat{n}_{\mathrm{S}}}-H_{0}^{(1)}(\underline{r}) \frac{\partial \hat{\eta}_{\mathrm{S}}^{(0)}\left(\vec{X}_{j}\right)}{\partial \hat{n}_{\mathrm{S}}}\right\} d S .
$$


Note that the outward normal directions of the boundary for forest subzone and open water are opposite to each other, i.e. $\hat{n}_{\xi}=-\hat{n}_{\mathrm{S}}$.

\subsection{Matching conditions along the boundaries}

As noted in Chang et al. (2016), the boundary of a forest subzone can be in contact with other subzones and/or with open water. Referring the former as the interface with other subzones and the latter as the exterior boundary, the continuity of wave amplitude and normal flux is required.

We first consider the exterior boundary of subzone $\xi$, i.e. $\mathrm{S}_{\xi}$. The continuity of wave amplitude and normal velocity spectrum respectively yields

$$
\hat{\eta}_{\xi}^{(0)}=\hat{\eta}_{\mathrm{I}}^{(0)}+\hat{\eta}_{\mathrm{S}}^{(0)} \quad \text { and } \quad-\mathrm{i} \hat{\mathcal{K}}_{\xi} \frac{\partial \hat{\eta}_{\xi}^{(0)}}{\partial \hat{n}_{\xi}}=\frac{\partial \hat{\eta}_{\mathrm{I}}^{(0)}}{\partial \hat{n}_{\xi}}+\frac{\partial \hat{\eta}_{\mathrm{S}}^{(0)}}{\partial \hat{n}_{\xi}}
$$

in which $\hat{\mathcal{K}} \rightarrow \mathrm{i}$ for the open water region has been applied. Note that in (3.7) the incident wave amplitude spectrum, i.e. $\hat{\eta}_{\mathrm{I}}^{(0)}=\hat{A}_{0} e^{\mathrm{i} X}$ with $\hat{A}_{0}=A_{s} / H_{\text {inc }}$, and the scattered wave amplitude spectrum $\hat{\eta}_{\mathrm{S}}^{(0)}$ have to be included within the open water region.

On the other hand, along the interface between subzone $\xi$ and its adjacent subzone $\psi$ (i.e. $\mathrm{D}_{\xi \psi}$ ), the wave amplitude and normal velocity spectrum have to be continuous:

$$
\hat{\eta}_{\xi}^{(0)}=\hat{\eta}_{\psi}^{(0)} \quad \text { and } \quad \hat{\mathcal{K}}_{\xi} \frac{\partial \hat{\eta}_{\xi}^{(0)}}{\partial \hat{n}_{\xi}}=-\hat{\mathcal{K}}_{\psi} \frac{\partial \hat{\eta}_{\psi}^{(0)}}{\partial \hat{n}_{\psi}} .
$$

Note that the negative sign reflects the opposite directions of $\hat{n}_{\xi}$ and $\hat{n}_{\psi}$.

\subsection{Numerical implementation}

In the numerical computations we discretize the boundary of a forest subzone $\xi$ with constant element of length $\Delta S$. Accordingly, (3.2) in discrete form becomes

$$
\hat{\eta}_{\xi}^{(0)}\left(\vec{X}_{i}\right)=\frac{-\mathrm{i} \pi}{2 \alpha} \sum_{j=1}^{\mathbb{N}_{\xi}}\left\{\hat{\eta}_{\xi}^{(0)}\left(\vec{X}_{j}\right) \frac{\partial\left[H_{0}^{(1)}(\hat{\beta} \underline{r})\right]}{\partial \hat{n}_{\xi}}-H_{0}^{(1)}(\hat{\beta} \underline{r}) \frac{\partial \hat{\eta}_{\xi}^{(0)}\left(\vec{X}_{j}\right)}{\partial \hat{n}_{\xi}}\right\} \Delta S
$$

in which $\vec{X}_{j}$ denotes the middle point of each boundary element (see Fig.2 in Chang et al. 2016 and also Fig.S2 in the Supplementary Materials). $\mathbb{N}_{\xi}$ represents the total number of elements on the boundary of subzone $\xi$, which can include the exterior boundary $\mathrm{S}_{\xi}$ and/or interfaces with other subzones $\mathrm{D}_{\xi \psi}$. The wave amplitude spectrum can also be expressed in terms of its normal derivatives in the following index notation formulation:

$$
\left(\hat{\eta}_{\xi}^{(0)}\right)_{i}=\left(\hat{\mathcal{G}}_{\xi}\right)_{i j}\left(\frac{\partial \hat{\eta}_{\xi}^{(0)}}{\partial \hat{n}_{\xi}}\right)_{j} \text { with } i, j=1 \cdots \mathbb{N}_{\xi}
$$

where the coefficient matrix $\left[\hat{\mathcal{G}}_{\xi}\right]=\left[\mathcal{I}+\hat{\mathcal{C}}_{\xi}\right]^{-1}\left[\hat{\mathcal{D}}_{\xi}\right]$ with $\mathcal{I}$ being the identity matrix while $\hat{C}_{\xi}$ and $\hat{D}_{\xi}$ are respectively defined as

$$
\left(\hat{\mathcal{C}}_{\xi}\right)_{i j}=\frac{\mathrm{i} \pi}{2 \alpha}\left[-\hat{\beta} H_{1}^{(1)}\left(\hat{\beta} \underline{r}_{i j}\right)\right] \frac{\partial \underline{r}_{i j}}{\partial \hat{n}_{\xi}} \Delta S, \quad\left(\hat{\mathcal{D}}_{\xi}\right)_{i j}=\frac{\mathrm{i} \pi}{2 \alpha} H_{0}^{(1)}\left(\hat{\beta} \underline{r}_{i j}\right) \Delta S .
$$

On the other hand, the wave amplitude spectrum for open water as shown in (3.5) also needs to be expressed in discrete form as

$$
\hat{\eta}_{\mathrm{S}}^{(0)}\left(\vec{X}_{i}\right)=\frac{-\mathrm{i} \pi}{2(2 \pi-\alpha)} \sum_{j=1}^{\mathbb{N}_{\mathrm{S}}}\left\{\hat{\eta}_{\mathrm{S}}^{(0)}\left(\vec{X}_{j}\right) \frac{\partial\left[H_{0}^{(1)}(\underline{r})\right]}{\partial \hat{n}_{\mathrm{S}}}-H_{0}^{(1)}(\underline{r}) \frac{\partial \hat{\eta}_{\mathrm{S}}^{(0)}\left(\vec{X}_{j}\right)}{\partial \hat{n}_{\mathrm{S}}}\right\} \Delta S
$$


where $\mathbb{N}_{S}$ denotes the total number of elements along the exterior boundary of all the forest patches, i.e. the boundary separating the forest region from open water $\left(S=\sum S_{\xi}\right)$. Likewise, we express the wave amplitude spectrum for open water in terms of its normal derivatives as

$$
\left(\hat{\eta}_{\mathrm{S}}^{(0)}\right)_{i}=\left(\hat{\mathcal{Q}}_{\mathrm{S}}\right)_{i j}\left(\frac{\partial \hat{\eta}_{\mathrm{S}}^{(0)}}{\partial \hat{n}_{\mathrm{S}}}\right)_{j} \quad \text { with } \quad i, j=1 \cdots \mathbb{N}_{\mathrm{S}}
$$

in which the coefficient matrix $\left[\hat{\mathcal{Q}}_{\mathrm{S}}\right]=\left[\mathcal{I}+\hat{\mathcal{E}}_{\mathrm{S}}\right]^{-1}\left[\hat{\mathcal{F}}_{\mathrm{S}}\right]$ with

$$
\left(\hat{\mathcal{E}}_{\mathrm{S}}\right)_{i j}=\frac{-\mathrm{i} \pi}{2(2 \pi-\alpha)} H_{1}^{(1)}\left(\underline{r}_{i j}\right) \frac{\partial \underline{r}_{i j}}{\partial \hat{n}_{\mathrm{S}}} \Delta S, \quad\left(\hat{\mathcal{F}}_{\mathrm{S}}\right)_{i j}=\frac{\mathrm{i} \pi}{2(2 \pi-\alpha)} H_{0}^{(1)}\left(\underline{r}_{i j}\right) \Delta S .
$$

As introduced in Sec.3.3, the matching conditions must be imposed for solving the wave amplitude spectrum along the boundaries, including both the interfaces and exterior boundaries. In (3.7), the continuity of wave and normal velocity spectrum along the exterior boundary $\mathrm{S}_{\xi}$ of a forest subzone respectively yields

$$
\left(\hat{\mathcal{G}}_{\xi}\right)_{i j}\left(\frac{\partial \hat{n}_{\xi}^{(0)}}{\partial \hat{n}_{\xi}}\right)_{j}=\left(\hat{A}_{0} e^{\mathrm{i} X_{1}}\right)_{i}+\left(\hat{\mathcal{Q}}_{\mathrm{S}}\right)_{i m}\left(\frac{\partial \hat{\eta}_{\mathrm{S}}^{(0)}}{\partial \hat{n}_{\mathrm{S}}}\right)_{m}
$$

and

$$
-\mathrm{i} \hat{\mathcal{K}}\left(\frac{\partial \hat{\eta}_{\xi}^{(0)}}{\partial \hat{n}_{\xi}}\right)_{i}=\hat{A}_{0}\left(\frac{\partial e^{\mathrm{i} X_{1}}}{\partial \hat{n}_{\xi}}\right)_{i}+\left(\frac{\partial \hat{\eta}_{\mathrm{S}}^{(0)}}{\partial \hat{n}_{\xi}}\right)_{i} .
$$

where the substitution of (3.10) and (3.13) has been made in (3.15). Assuming the incident waves in $X_{1}$ direction, $X_{1 i}$ in (3.15) and (3.16) denotes the $X_{1}$ coordinate of point $i$. Note that $\xi$ in (3.15) and (3.16) can be any subzone that is exposed to the open water region such that $\vec{X}_{i}$ is located on the exterior boundary of subzone $\xi$. In (3.15), $\vec{X}_{j} \in \mathrm{S}_{\xi}+\mathrm{D}_{\xi \psi}$ with $j=1 \cdots \mathbb{N}_{\xi}$ denotes the boundary points of subzone $\xi$ while $\vec{X}_{m} \in \mathrm{S}$ with $m=1 \cdots \mathbb{N}_{\mathrm{S}}$ is on the boundary of open water.

Along the interfaces between subzones, (3.8) also requires the continuity of wave amplitude and normal velocity spectrum such that

$$
\left(\hat{\mathcal{G}}_{\xi}\right)_{i j}\left(\frac{\partial \hat{\eta}_{\xi}^{(0)}}{\partial \hat{n}_{\xi}}\right)_{j}=\left(\hat{\mathcal{G}}_{\psi}\right)_{i k}\left(\frac{\partial \hat{\eta}_{\psi}^{(0)}}{\partial \hat{n}_{\psi}}\right)_{k}
$$

and

$$
\hat{\mathcal{K}}_{\xi}\left(\frac{\partial \hat{\eta}_{\xi}^{(0)}}{\partial \hat{n}_{\xi}}\right)_{i}=-\hat{\mathcal{K}}_{\psi}\left(\frac{\partial \hat{\eta}_{\psi}^{(0)}}{\partial \hat{n}_{\psi}}\right)_{i}
$$

in which $\vec{X}_{j}\left(j=1 \cdots \mathbb{N}_{\xi}\right)$ and $\vec{X}_{k}\left(k=1 \cdots \mathbb{N}_{\psi}\right)$ represent the boundary points of subzones $\xi$ and $\psi$, respectively. Also note the outward normal directions along the interface with respect to these two adjacent subzones $(\xi$ and $\psi)$ are opposite, i.e. $\hat{n}_{\xi}=-\hat{n}_{\psi}$.

With (3.15), (3.16), (3.17) and (3.18), a system of equations is formulated for solving the leading-order wave amplitude spectrum $\hat{\eta}_{\xi}^{(0)}, \hat{\eta}_{\psi}^{(0)}$ and $\hat{\eta}_{\mathrm{S}}^{(0)}$ as well as their normal derivatives along the exterior boundaries of forest patches and the interfaces between subzones. Once the solutions along boundaries are known, the wave amplitude spectrum within a subzone and the open water region can be readily obtained from (3.3) and (3.6), respectively. As noted previously, the wave amplitude spectrums $\hat{\eta}_{\xi}^{(0)}$ and $\hat{\eta}_{\mathrm{S}}^{(0)}$ are solved independently for each harmonic. The free surface elevation can then be obtained by the inverse transform in (2.14). In the numerical computations, the infinite range of harmonics $\omega$ needs to be truncated to a finite number. It has been found that the harmonics ranging from $10^{-3}$ to 10 (i.e. $10^{-3} \leq \omega \leq 10$ ) with 40 equally-distributed terms in total is sufficient to reconstruct the shape of incident soliton in (2.11). Therefore, we start with this arrangement in the numerical computations and perform convergent tests. According to the results, it shows that this arrangement is able to bring about convergent free surface elevations for small amplitude waves. For relatively larger waves, however, the range of harmonic components needs to be modified and higher values have to be included. The details for each forest configuration will be presented. 
An example sketching the discretization of the forest boundary has been provided in Fig.2 of Chang et al. (2016) (also presented as Fig.S2 in the Supplementary Materials). The definition of interior angle and element length are presented in the same figure. The outward normal directions along the boundaries of subzones and of open water can also be found. The direction of integration along the boundary of subzones is specified as well. Similar to Chang et al. (2016), a computer program named CHEWAVE-T (Cornell HomogEnization model on WAve-VEgetation interaction-Transient wave) is also developed on the basis of the present approach. Applying Fourier transform, the model is able to solve the propagation of a transient long wave through a general forest region which has multiple forest patches of arbitrary shape. The numerical solver on the micro-scale problem is also provided. Inputs for the numerical computations, such as the conditions of incident soliton, the forest properties and the discretization of forest boundary, are briefly described in Appendix A. Comparing the present model for transient waves with that for periodic waves in Chang et al. (2016), both models share the integral formulations for the Helmholtz equation but with different contributions from the respective cell problems. As mentioned in Sec.3.1 and 3.2, the wave amplitude spectrum for each harmonic is solved separately.

\section{Model validation and numerical results}

The infinitely long forest belt studied in Mei et al. (2011) is first examined to validate the present approach. The homogeneous circular forest presented in Liu et al. (2015) and Chang et al. (2016) (for studying periodic waves) is then used to check the numerical model. The analytical solutions for both special forest configurations are provided. Negligible differences between the numerical results and the analytical solutions are observed, and hence the detailed comparison will not be shown here. The comparison between experimental data and model results is, however, provided. To further investigate and validate the present model, the experiments presented in Maza (2015) and Maza et al. (2016) on multiple circular patches are used as another special forest configuration. Numerically-simulated free surfaces elevation compared with the experimental measurements are presented herein.

\subsection{Solitary wave through an infinitely long forest belt}

Considering a transient long wave with a soliton-like shape propagating through a forest belt with a finite thickness (i.e. $0<X<L_{\mathrm{F}},-\infty<Y<\infty$ ), the analytical solution of wave amplitude spectrum is shown first. The comparison with experimental data reported in Mei et al. (2011) is provided. The nonlinearity effect on the wave attenuation is also discussed.

\subsubsection{Analytical solution}

The leading-order wave amplitude spectrum inside the forest (with subscript F) can be obtained by solving (2.28):

$$
\hat{\eta}_{\mathrm{F}}^{(0)}=C_{1} e^{\gamma X}+C_{2} e^{-\gamma X} \quad, \quad \gamma=\mathrm{i} \hat{\beta}=\sqrt{-\mathrm{i} n / \hat{\mathcal{K}}}
$$

The cell-averaged leading-order velocity spectrum can then be derived from (2.24):

$$
\left\langle\hat{u}_{\mathrm{F}}^{(0)}\right\rangle=-\hat{\mathcal{K}} \frac{\partial \hat{\eta}_{\mathrm{F}}^{(0)}}{\partial X}=-\gamma \hat{\mathcal{K}}\left[C_{1} e^{\gamma X}-C_{2} e^{-\gamma X}\right]
$$

where $C_{1}$ and $C_{2}$ are undetermined coefficients. In open water, the solutions in the incidence region (i.e. $X<0)$ are

$$
\hat{\eta}_{\mathrm{I}}^{(0)}+\hat{\eta}_{\mathrm{R}}^{(0)}=\hat{A}_{0} e^{\mathrm{i} X}+\hat{A}_{0} R e^{-\mathrm{i} X}, \quad\left\langle\hat{u}_{\mathrm{I}}^{(0)}\right\rangle+\left\langle\hat{u}_{\mathrm{R}}^{(0)}\right\rangle=\hat{A}_{0} e^{\mathrm{i} X}-\hat{A}_{0} R e^{-\mathrm{i} X}
$$

while in the transmission region (i.e. $X>L_{\mathrm{F}}$ ) the solutions become

$$
\hat{\eta}_{\mathrm{T}}^{(0)}=\hat{A}_{0} T e^{\mathrm{i} X}, \quad\left\langle\hat{u}_{\mathrm{T}}^{(0)}\right\rangle=\hat{A}_{0} T e^{\mathrm{i} X}
$$

where $|R|$ and $|T|$ respectively represent the reflection and transmission coefficients of each harmonic in the transformed domain. Note that $\hat{A}_{0}=A_{s} / H_{\text {inc }}$ with $A_{s}(\omega)$ defined in (2.13) and $H_{\text {inc }}$ the incident wave 
height. The unknown coefficients $C_{1}, C_{2}, R$ and $T$ are to be determined by requiring the wave amplitude and velocity spectrum to be continuous along the edges of the forest:

$$
\hat{\eta}_{\mathrm{F}}^{(0)}=\hat{\eta}_{\mathrm{I}}^{(0)}+\hat{\eta}_{\mathrm{R}}^{(0)}, \quad\left\langle\hat{u}_{\mathrm{F}}^{(0)}\right\rangle=\left\langle\hat{u}_{\mathrm{I}}^{(0)}\right\rangle+\left\langle\hat{u}_{\mathrm{R}}^{(0)}\right\rangle \quad \text { along } \quad X=0
$$

and

$$
\hat{\eta}_{\mathrm{F}}^{(0)}=\hat{\eta}_{\mathrm{T}}^{(0)}, \quad\left\langle\hat{u}_{\mathrm{F}}^{(0)}\right\rangle=\left\langle\hat{u}_{\mathrm{T}}^{(0)}\right\rangle \quad \text { along } \quad X=L_{\mathrm{F}} .
$$

Substituting the wave amplitude and velocity spectrum into these two conditions (4.5) and (4.6), the coefficients are derived as

$$
C_{1}=\frac{2 \hat{A}_{0}(1-\gamma \hat{\mathcal{K}})}{(1-\gamma \hat{\mathcal{K}})^{2}-(1+\gamma \hat{\mathcal{K}})^{2} e^{2 \gamma L_{\mathrm{F}}}}, \quad C_{2}=\frac{-2 \hat{A}_{0}(1+\gamma \hat{\mathcal{K}}) e^{2 \gamma L_{\mathrm{F}}}}{(1-\gamma \hat{\mathcal{K}})^{2}-(1+\gamma \hat{\mathcal{K}})^{2} e^{2 \gamma L_{\mathrm{F}}}}
$$

and

$$
R=\frac{2(1-\gamma \hat{\mathcal{K}})-2(1+\gamma \hat{\mathcal{K}}) e^{2 \gamma L_{\mathrm{F}}}}{(1-\gamma \hat{\mathcal{K}})^{2}-(1+\gamma \hat{\mathcal{K}})^{2} e^{2 \gamma L_{\mathrm{F}}}}-1, \quad T=\frac{-4 \gamma \hat{\mathcal{K}} e^{(\gamma-\mathrm{i}) L_{\mathrm{F}}}}{(1-\gamma \hat{\mathcal{K}})^{2}-(1+\gamma \hat{\mathcal{K}})^{2} e^{2 \gamma L_{\mathrm{F}}}} .
$$

With the wave amplitude spectrum for each region, the free surface elevation can then be obtained by the inverse Fourier transform in (2.14).

\subsubsection{Numerical results}

To check the approach, we compare the analytical solutions in the previous section with the experimental data reported by Mei et al. (2011). The experimental setup and the wave conditions can be found in Mei et al. (2011)'s Fig.8 and Table 5, respectively. The model forest has $1.08 \mathrm{~m}$ in width. As stated in Sec.3.4, in the computations we start with the truncated range of harmonics as $10^{-3} \leq \omega \leq 10$ where totally 40 equally-distributed values are included. To test the numerical results, here we modify the range of harmonics by increasing the highest value by one for each time and compute the relative error of the maximum wave heights obtained from two successive arrangements. The criterion for convergence requires the error to be less than $0.1 \%$. It is shown that this range (i.e. $10^{-3} \leq \omega \leq 10$ ) provides convergent results for the cases with relatively smaller waves (e.g. $H_{\mathrm{inc}} / h_{0}=0.0413$ and 0.0744 ). However, for the cases with larger waves (e.g. $H_{\text {inc }} / h_{0}=0.1113,0.1487,0.1864$ ), we need to adopt $10^{-3} \leq \omega \leq 15$ with 40 equally-distributed values to have convergent free surface elevation. For both ranges, the small parameter in (2.8) is still satisfied. Comparing with the data at the end of the forest (i.e. wave gauge 6 in Mei et al. 2011's Fig.8), the relative errors of numerical results for all the reported cases are always less than $10 \%$. Two wave conditions are selected to present the comparison between the predicted free surface elevation and the experimental measurements in Fig.1. In addition, defining the damping rate as

$$
\Delta H^{*}(\%)=\frac{\left|H_{\text {end }}-H_{\text {inc }}\right|}{H_{\text {inc }}} \times 100 \%
$$

where $H_{\text {end }}$ denotes the wave height measured at wave gauge 6 , the comparison of different cases (with different $H_{\text {inc }} / h_{0}$ ) is presented in Fig.2. When the nonlinearity (i.e. $H_{\text {inc }} / h_{0}$ ) becomes larger, the prediction starts to overestimate the wave attenuation by the forest. In (2.9), the eddy viscosity $\nu_{e}$, which directly affects the dissipation rate, is approximately linear with the incident wave nonlinearity when $H_{\text {inc }} / h$ is small enough, i.e. $\mathcal{O}\left(H_{\mathrm{inc}} / h_{0}\right) \ll 1$. This may explain the numerical damping rates and the reported data have different behaviors when the nonlinearity of incident waves becomes higher. The limitation of present linear model also contributes to the discrepancy between numerical results and measurements, which may not be clear here due to the lack of cases with higher nonlinearity. Further discussions on the nonlinearity of incident waves will be presented later for different forest configurations. It is also observed that the forest has higher efficiency for damping larger waves. In addition to the wave crest attenuation, the reflection by the forest belt is also detected by the present model at wave gauge 2, where the reflected wave height ranges from $15 \%$ to $20 \%$ of the incident wave. 


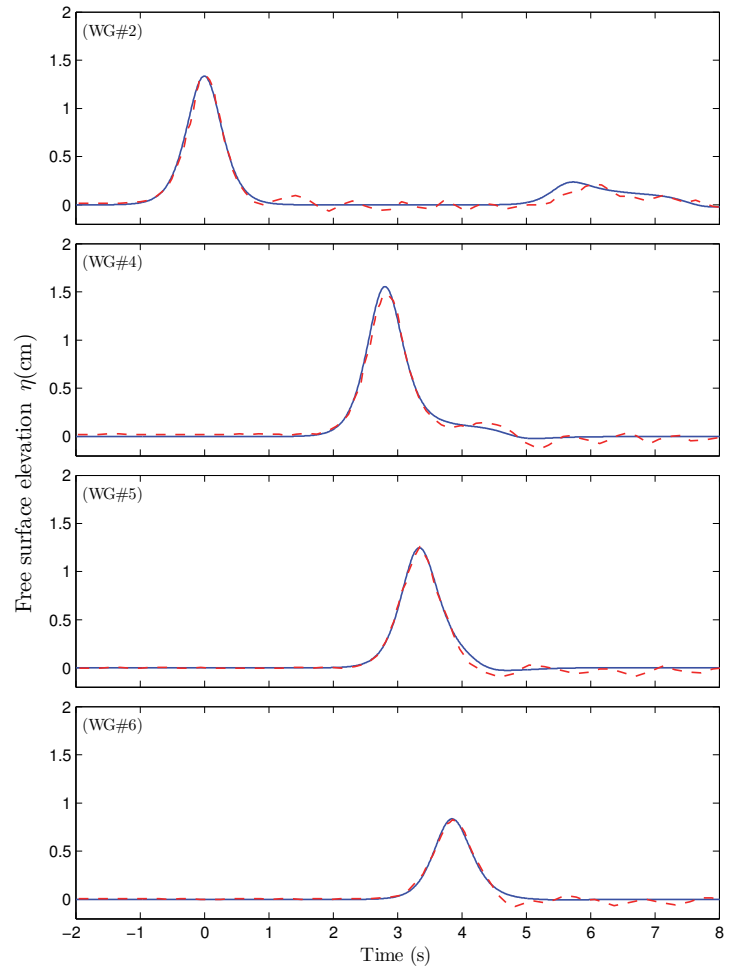

(a) $H_{\text {inc }}=1.34 \mathrm{~cm}$
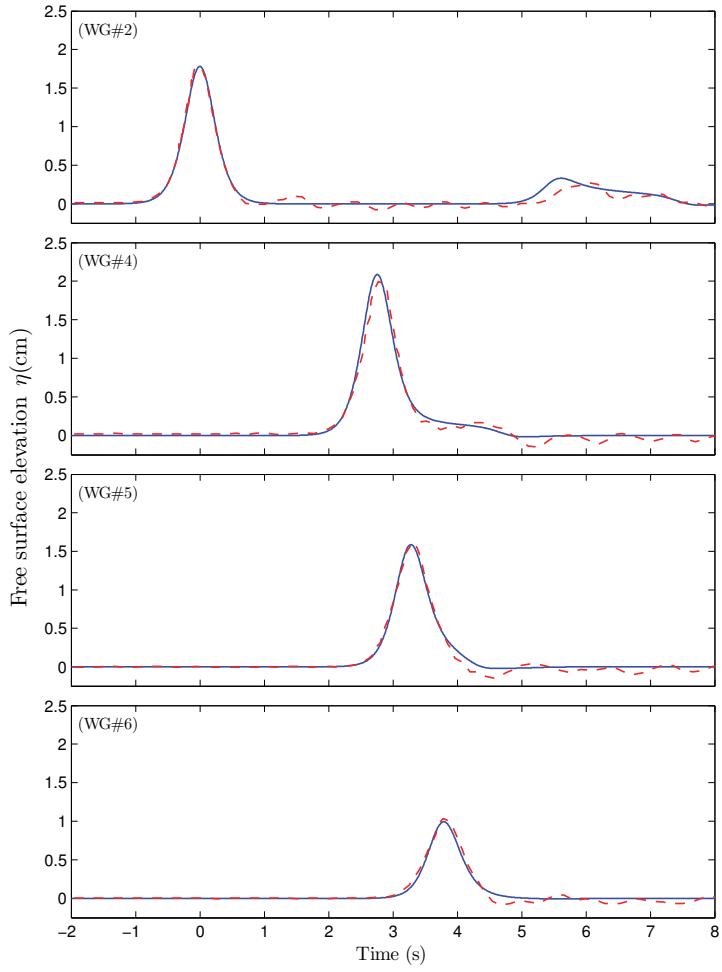

(b) $H_{\text {inc }}=1.78 \mathrm{~cm}$

Figure 1: Wave height comparison between model results (blue solid lines) and experimental data (red dashed lines) on the forest belt. For both cases, the water depth $h_{0}=12 \mathrm{~cm}$, the width of the forest region $L_{\mathrm{F}}=1.08 \mathrm{~m}$ and the cylinder size $d=1 \mathrm{~cm}$ with porosity $n \approx 91.27 \%$.

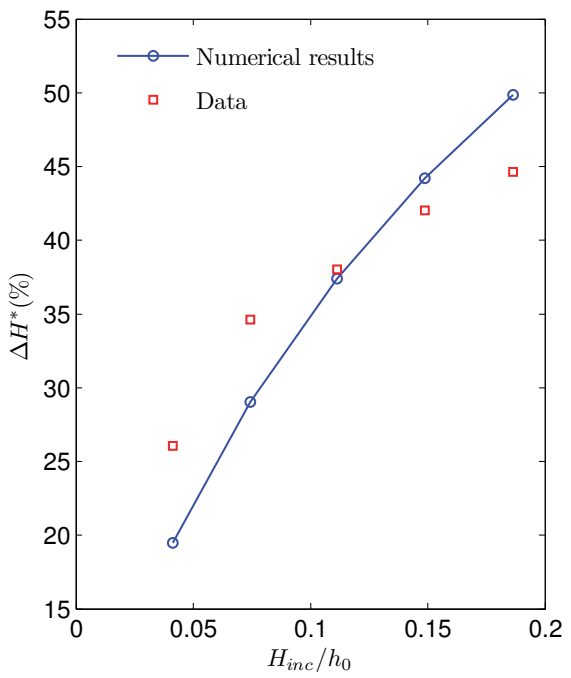

Figure 2: Comparison of damping rate as defined in (4.9): circles show the model results and squares represent the experimental measurements. 


\subsection{Solitary wave though a circular forest}

To check the present numerical model, we consider a circular forest with radius $R$, which was studied in Liu et al. (2015) and Chang et al. (2016) for periodic waves. The laboratory experiments were conducted in the Directional Wave Basin at the University of Cantabria in Spain. The experimental setup and the detailed arrangement of wave gauges have been given in Liu et al. (2015)'s Fig.7. Two water depths with different wave conditions are tested (Table 1 and 2). In the following, we first present the analytical solutions for wave amplitude spectrum. The numerical results obtained by the present model agree very well with the analytical solutions and the comparison will not be shown here. Instead, the comparison between the model simulations and experimental data will be presented and discussed.

\subsubsection{Analytical solution}

The governing equation (2.28) for wave amplitude spectrum within the forest region can be expressed in cylindrical coordinates as

$$
\frac{1}{r} \frac{\partial}{\partial r}\left(r \frac{\partial \hat{\eta}_{\mathrm{F}}^{(0)}}{\partial r}\right)+\frac{1}{r^{2}} \frac{\partial^{2} \hat{\eta}_{\mathrm{F}}^{(0)}}{\partial \theta^{2}}+\hat{\beta}^{2} \hat{\eta}_{\mathrm{F}}^{(0)}=0, \quad \hat{\beta}=\sqrt{\frac{\mathrm{in}}{\hat{\mathcal{K}}}}
$$

where the analytical solution can be readily obtained as

$$
\hat{\eta}_{\mathrm{F}}^{(0)}=\hat{A}_{0} \sum_{m=0}^{\infty} \epsilon_{m} \mathrm{i}^{m} \cos m \theta C_{m} J_{m}(\hat{\beta} r) .
$$

For the open water region, the wave amplitude spectrum, including the incident and scattered waves, gives

$$
\hat{\eta}_{\mathrm{I}}^{(0)}+\hat{\eta}_{\mathrm{S}}^{(0)}=\hat{A}_{0} \sum_{m=0}^{\infty} \epsilon_{m} \mathrm{i}^{m} \cos m \theta\left[J_{m}(r)+B_{m} H_{m}^{(1)}(r)\right]
$$

where $\hat{\mathcal{K}}=\mathrm{i}$ and $n=1$ have been applied. In (4.12), $J_{m}$ and $H_{m}$ denote respectively the Bessel function of the first kind and the Hankel function of the first kind. The Jacobi symbol has the following definition: $\epsilon_{m}$ has $\epsilon_{0}=1$ and $\epsilon_{m}=2$ when $m \geq 1$. The unknown coefficients $B_{m}$ and $C_{m}$ are determined by the matching conditions at $r=R$ :

$$
\hat{\eta}_{\mathrm{F}}^{(0)}=\hat{\eta}_{\mathrm{I}}^{(0)}+\hat{\eta}_{\mathrm{S}}^{(0)} \quad \text { and } \quad-\mathrm{i} \hat{\mathcal{K}} \frac{\partial \hat{\eta}_{\mathrm{F}}^{(0)}}{\partial r}=\frac{\partial \hat{\eta}_{\mathrm{I}}^{(0)}}{\partial r}+\frac{\partial \hat{\eta}_{\mathrm{S}}^{(0)}}{\partial r} .
$$

Substituting (4.11) and (4.12) into (4.13) yields

$$
B_{m}=\frac{-\Delta J /\left(\mathrm{i} \hat{\beta} \hat{\mathcal{K}} \Delta J_{\hat{\beta}}\right)-J_{m}(R) / J_{m}(\hat{\beta} R)}{H_{m}^{(1)}(R) / J_{m}(\hat{\beta} R)+2 \Delta H^{(1)} /\left(\mathrm{i} \hat{\beta} R \hat{\mathcal{K}} \Delta J_{\hat{\beta}}\right)} \quad \text { and } \quad C_{m}=\frac{\Delta J+2 B_{m} \Delta H^{(1)} / R}{-\mathrm{i} \hat{\beta} \hat{\mathcal{K}} \Delta J_{\hat{\beta}}}
$$

where $\Delta J=\left[J_{m-1}(R)-J_{m+1}(R)\right], \Delta J_{\hat{\beta}}=\left[J_{m-1}(\hat{\beta} R)-J_{m+1}(\hat{\beta} R)\right]$ and $\Delta H^{(1)}=\left[m H_{m}^{(1)}(R)-R H_{m+1}^{(1)}(R)\right]$. The solutions for the free surface elevation can be obtained by the inverse Fourier transform of the wave amplitude spectrum.

\subsubsection{Numerical results}

In the present numerical computations, the circular forest boundary is discretized as shown in Chang et al. (2016)'s Fig.4 (also shown as Fig.S4 in the Supplementary Materials). For each harmonic, we adopt the same discretization with 180 boundary elements $\left(\mathbb{N}_{\xi}=\mathbb{N}_{S}=180\right)$ in total. This was confirmed by a convergence test in Chang et al. (2016). We first compare the numerical results with the analytical solutions shown in the previous Sec.4.2.1. The agreements are excellent. Thus, the detailed comparison is skipped.

In this section we shall compare the numerical results with the experimental measurements. Two cases with lowest values of $H_{\text {inc }} / h_{0}$ are first shown in Fig.3 and Fig.4. On the other hand, Fig.5 and Fig.6 show the comparisons of two other cases with higher values of $H_{\mathrm{inc}} / h_{0}$. Comparisons are made at gauges along 
the centerline $(Y=0)$ as well as the off-centerline $(Y=-R / 2)$. The wave crest attenuation is evident in both sets of cases. The wave height in front of the forest increases slightly due to the wave scattering and reflection. In addition, the diffracted wave converging behind the forest is also evident at wave gauge 19 and 20, resulting in a slightly increased wave height. The wave height variations along the centerline of the forest are shown in Fig.7. Overall, the numerically-simulated wave heights agree well with the measured wave heights. The relative differences are always below $17 \%$. More specifically, for the cases with smaller nonlinearity (i.e. $H_{\text {inc }} / h_{0} \leq 0.2$ ), the relative error is lower than $10 \%$.

Further investigation is also conducted on the damping rate along the centerline of the circular forest, which is defined as (4.9) with $H_{\text {end }}$ representing $H_{19}$, i.e. the measured height by wave gauge 19. Fig.8 shows the variation of the damping rate along the centerline with the nonlinearity of incident wave. Obviously, the relative differences between the predicted and measured damping rate are below $5 \%$ when $H_{\mathrm{inc}} / h_{0} \leq 0.3$. On the other hand, the discrepancy becomes noticeable when the nonlinearity is beyond 0.3 , which confirms the limitation of present linear model. It is also shown that the forest is more efficient on damping larger waves.

It should be noted that during the calibration tests for the experiments (i.e. test runs without cylinders) wave gauge 10 shows irregularities. Namely, the wave height measurements at gauge 10 are approximately +5 to $+10 \%$ of the incident wave height for the wave tests in Table 1. On the other hand, for the wave conditions shown in Table 2, wave height measurements at gauge 10 are approximately -5 to $-8 \%$ of the incident wave height during the calibration tests.

Table 1: Experimental conditions and corresponding dimensionless parameters - Solitary Test I.

\begin{tabular}{c|c|ccccccc}
\hline Case & $h_{0}(\mathrm{~cm})$ & $H_{\text {inc }}(\mathrm{cm})$ & $k_{s}(1 / \mathrm{m})$ & $L(\mathrm{~m})$ & $k_{s} h_{0}$ & $k_{s} H_{\text {inc }}$ & $H_{\text {inc }} / h_{0}$ & $\nu_{e}\left(\mathrm{~m}^{2} / \mathrm{s}\right)$ \\
\hline 3S1 & & 3.54 & 0.9916 & 6.3362 & 0.2975 & 0.0351 & 0.1180 & $2.1101 \mathrm{E}-04$ \\
3S2 & & 4.42 & 1.1081 & 5.6705 & 0.3324 & 0.0490 & 0.1473 & $2.6007 \mathrm{E}-04$ \\
3S3 & & 5.35 & 1.2191 & 5.1541 & 0.3657 & 0.0652 & 0.1783 & $3.1063 \mathrm{E}-04$ \\
3S4 & \multirow{3}{*}{30} & 7.34 & 1.4279 & 4.4003 & 0.4284 & 0.1048 & 0.2447 & $4.1466 \mathrm{E}-04$ \\
3S5 & & 8.32 & 1.5202 & 4.1330 & 0.4561 & 0.1265 & 0.2773 & $4.6397 \mathrm{E}-04$ \\
3S6 & & 9.21 & 1.5995 & 3.9283 & 0.4798 & 0.1473 & 0.3070 & $5.0774 \mathrm{E}-04$ \\
3S7 & & 10.92 & 1.7416 & 3.6076 & 0.5225 & 0.1902 & 0.3640 & $5.8930 \mathrm{E}-04$ \\
3S8 & & 11.26 & 1.7686 & 3.5527 & 0.5306 & 0.1991 & 0.3753 & $6.0514 \mathrm{E}-04$ \\
\hline
\end{tabular}

Table 2: Experimental conditions and corresponding dimensionless parameters - Solitary Test II.

\begin{tabular}{c|c|ccccccc}
\hline Case & $h_{0}(\mathrm{~cm})$ & $H_{\text {inc }}(\mathrm{cm})$ & $k_{s}(1 / \mathrm{m})$ & $L(\mathrm{~m})$ & $k_{s} h_{0}$ & $k_{s} H_{\text {inc }}$ & $H_{\text {inc }} / h_{0}$ & $\nu_{e}\left(\mathrm{~m}^{2} / \mathrm{s}\right)$ \\
\hline $4 \mathrm{~S} 1$ & & 3.58 & 0.6477 & 9.7006 & 0.2591 & 0.0232 & 0.0895 & $1.8721 \mathrm{E}-04$ \\
$4 \mathrm{~S} 2$ & & 4.54 & 0.7294 & 8.6141 & 0.2918 & 0.0331 & 0.1135 & $2.3483 \mathrm{E}-04$ \\
$4 \mathrm{~S} 3$ & & 5.44 & 0.7984 & 7.8694 & 0.3194 & 0.0434 & 0.1360 & $2.7859 \mathrm{E}-04$ \\
$4 \mathrm{~S} 4$ & \multirow{4}{*}{40} & 7.42 & 0.9325 & 6.7381 & 0.3730 & 0.0692 & 0.1855 & $3.7197 \mathrm{E}-04$ \\
$4 \mathrm{~S} 5$ & & 8.40 & 0.9922 & 6.3329 & 0.3969 & 0.0833 & 0.2099 & $4.1681 \mathrm{E}-04$ \\
$4 \mathrm{~S} 6$ & & 9.44 & 1.0518 & 5.9738 & 0.4207 & 0.0993 & 0.2359 & $4.6346 \mathrm{E}-04$ \\
$4 \mathrm{~S} 7$ & & 11.39 & 1.1553 & 5.4385 & 0.4621 & 0.1316 & 0.2848 & $5.4848 \mathrm{E}-04$ \\
$4 \mathrm{~S} 8$ & & 11.61 & 1.1664 & 5.3867 & 0.4666 & 0.1354 & 0.2903 & $5.5789 \mathrm{E}-04$ \\
\hline
\end{tabular}

The above $h_{0}$ represents the constant water depth and the incident wave height $H_{\text {inc }}$ is obtained from the averaged wave height measured by wave gauges 1,2 and $3 . k_{s}$ denotes the effective wavenumber defined in $(2.12)$ and the wave length $L=2 \pi / k_{s}$. For all the cases, the diameter of the circular forest is $3 \mathrm{~m}$ and the cell porosity is approximately $91.27 \%$. 

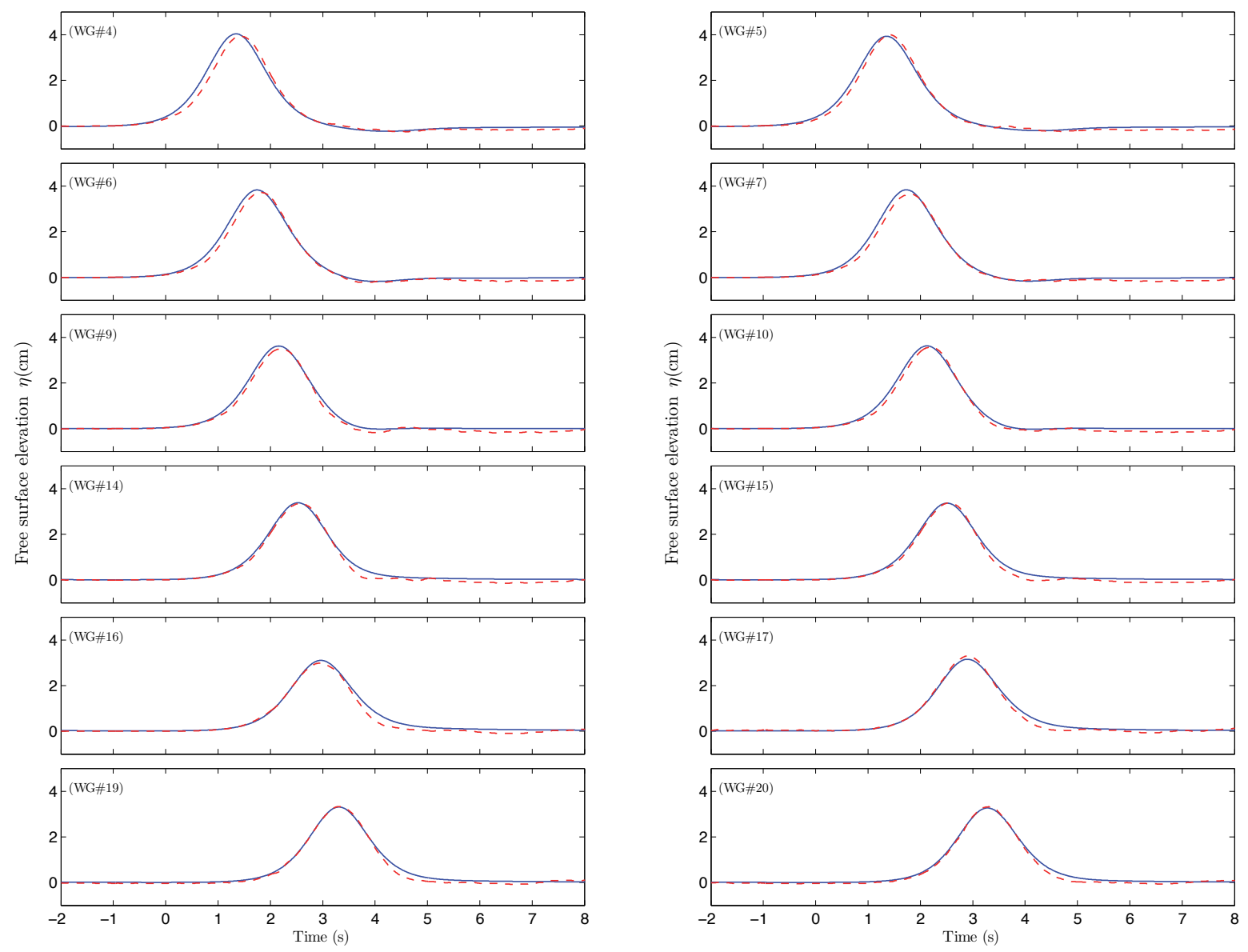

(a) $Y=0$ : along $\mathrm{WG} \# 4,6,9,14,16,19$

(b) $Y=-R / 2$ : along WG \#5, 7, 10, 15, 17, 20

Figure 3: Comparison of wave heights by numerical simulations (solid lines) with experimental data (dashed lines) for Case $4 \mathrm{~S} 1$. 

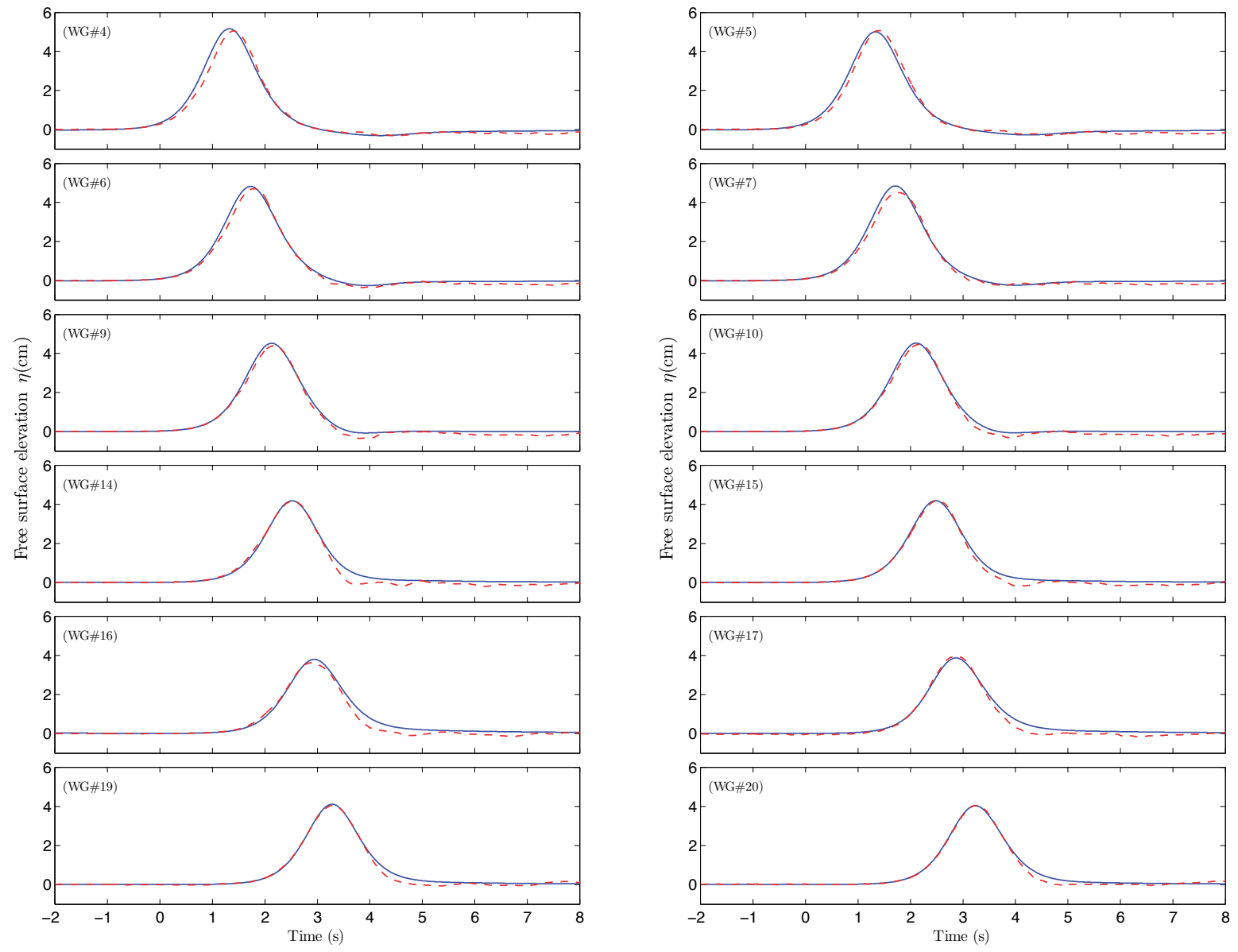

(a) $Y=0$ : along WG \#4, 6, 9, 14, 16, 19

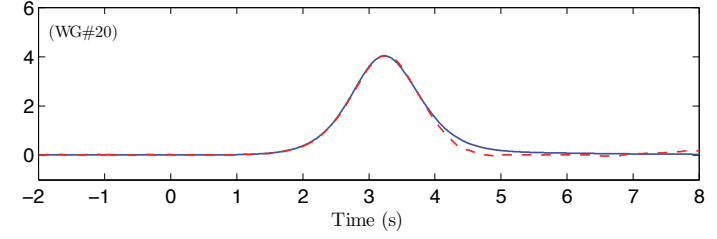

(b) $Y=-R / 2$ : along $\mathrm{WG} \# 5,7,10,15,17,20$

Figure 4: Comparison of wave heights by numerical simulations (solid lines) with experimental data (dashed lines) for Case $4 \mathrm{~S} 2$. 

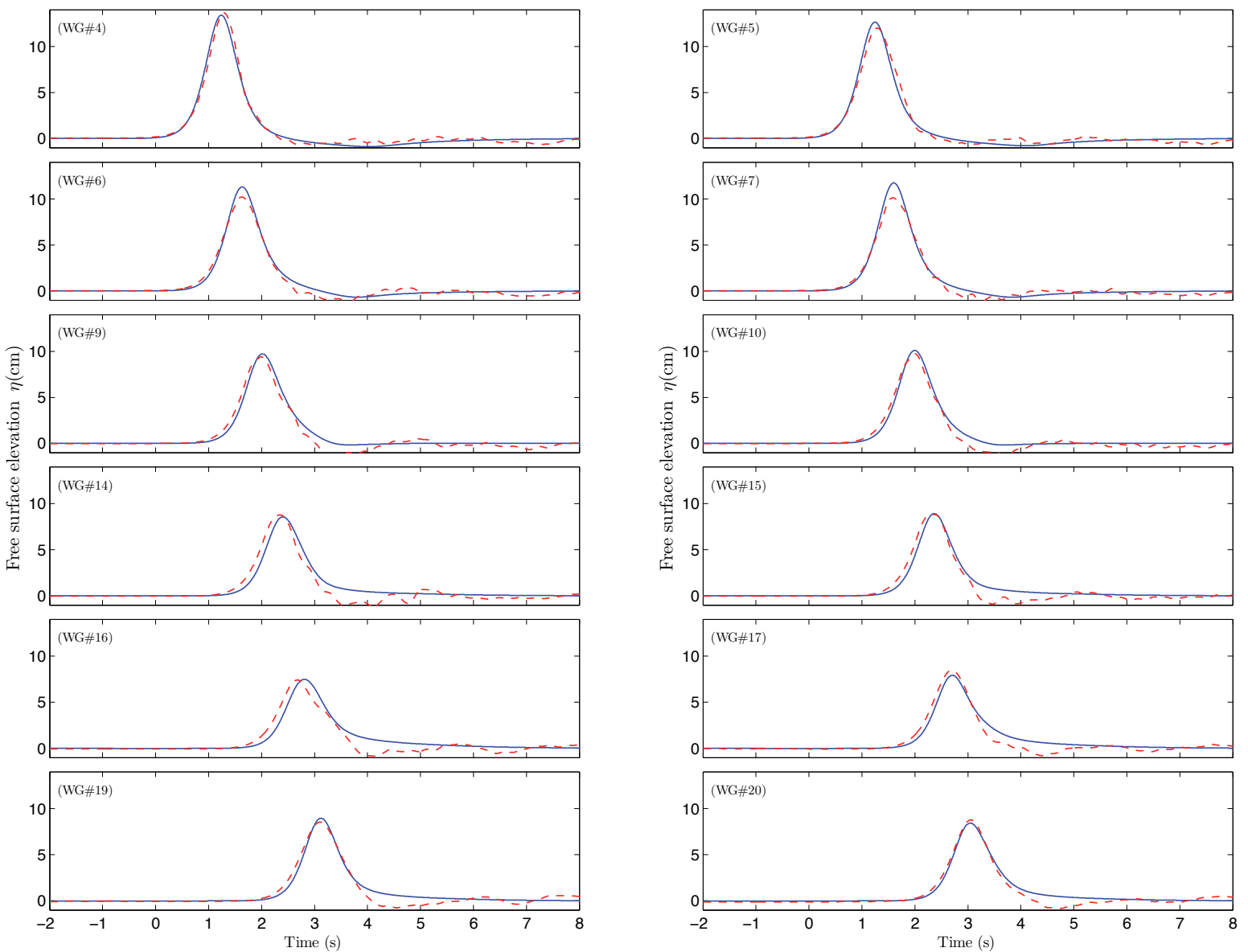

(a) $Y=0$ : along WG \#4, 6, 9, 14, 16, 19

(b) $Y=-R / 2$ : along $\mathrm{WG} \# 5,7,10,15,17,20$

Figure 5: Comparison of wave heights by numerical simulations (solid lines) with experimental data (dashed lines) for Case $4 \mathrm{~S} 7$. 

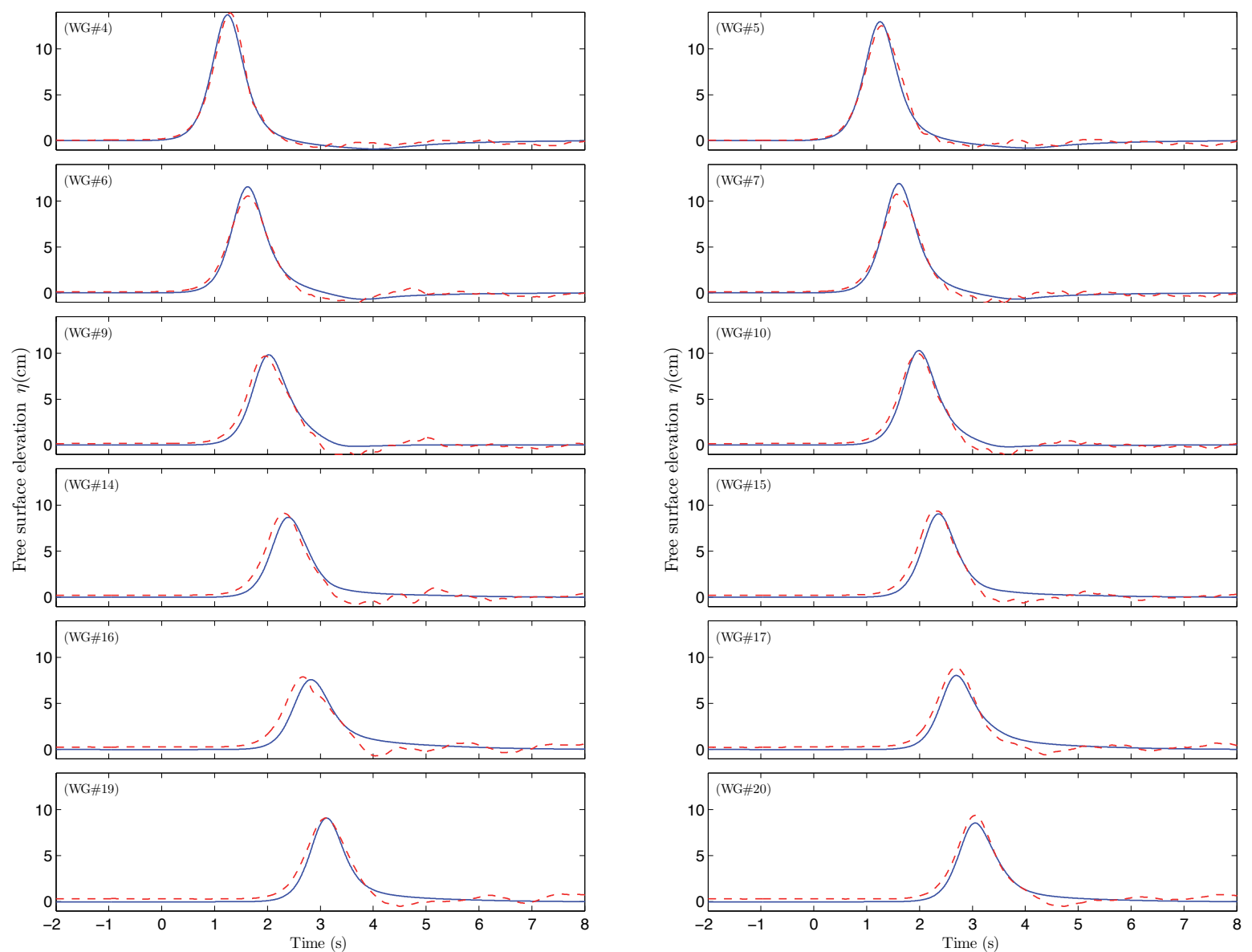

(a) $Y=0$ : along WG \#4, 6, 9, 14, 16, 19

(b) $Y=-R / 2$ : along $\mathrm{WG} \# 5,7,10,15,17,20$

Figure 6: Comparison of wave height by numerical simulations (solid lines) with experimental data (dashed lines) for Case $4 \mathrm{~S} 8$. 

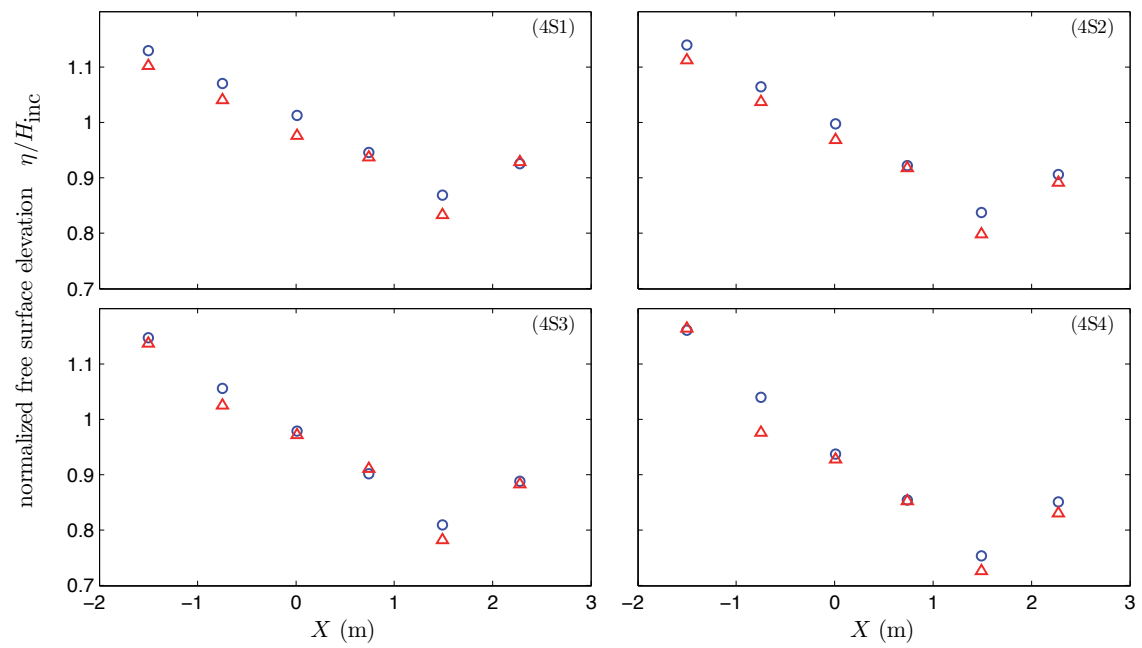

(a) $4 \mathrm{~S} 1-4 \mathrm{~S} 4$
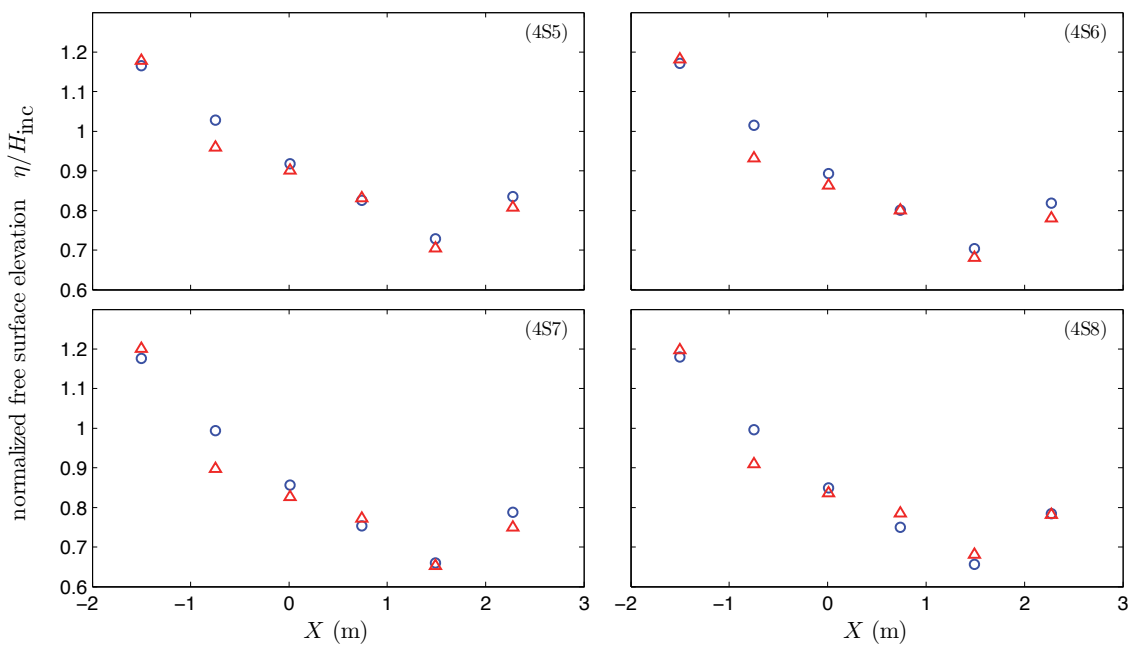

(b) $4 \mathrm{~S} 5-4 \mathrm{~S} 8$

Figure 7: Comparison of simulated and measured wave height profiles along the centerline for cases in Table 2: circles - model results; triangles - data. 


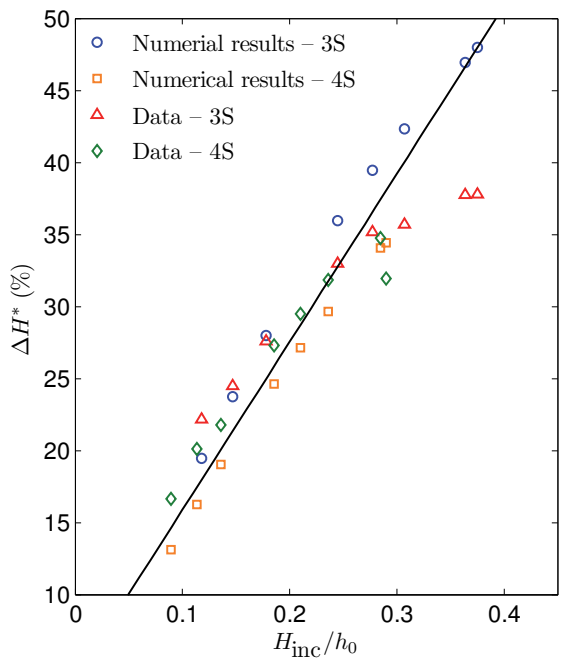

Figure 8: Comparison of damping rate along the centerline. $3 \mathrm{~S}$ and $4 \mathrm{~S}$ represent the wave conditions in Table 1 and 2 respectively. Linear fitting (solid) line for numerical simulation is $\Delta H^{*}(\%)=166.66\left(H_{\mathrm{inc}} / h_{0}\right)+4.22$ with $R^{2}=0.9541$.

\subsection{Solitary wave though multiple circular forest patches}

To further test the present numerical model, we employ one of Maza et al. (2016)'s experiments with multiple circular patches as another special forest configuration. The detailed experimental setup has been provided in Chang et al. (2016)'s Fig.3, which is used for studying periodic waves. The same figure is also provided in the Supplementary Materials (Fig.S3). Note that the experimental facility is the same as that presented in Sec.4.2 but with different arrangement of wave gauges. The experimental conditions can be found in Table 3.

Table 3: Experimental conditions and corresponding dimensionless parameters for multiple circular patches.

\begin{tabular}{c|c|ccccccc}
\hline Case & $h_{0}(\mathrm{~cm})$ & $H_{\text {inc }}(\mathrm{cm})$ & $k_{s}(1 / \mathrm{m})$ & $L(\mathrm{~m})$ & $k_{s} h_{0}$ & $k_{s} H_{\text {inc }}$ & $H_{\text {inc }} / h_{0}$ & $\nu_{e}\left(\mathrm{~m}^{2} / \mathrm{s}\right)$ \\
\hline 1 & & 4.59 & 1.1292 & 5.5645 & 0.3387 & 0.0518 & 0.1530 & $2.6941 \mathrm{E}-04$ \\
2 & 30 & 6.98 & 1.3924 & 4.5124 & 0.4177 & 0.0972 & 0.2327 & $3.9623 \mathrm{E}-04$ \\
3 & & 9.16 & 1.5951 & 3.9390 & 0.4785 & 0.1461 & 0.3053 & $5.0531 \mathrm{E}-04$ \\
\hline
\end{tabular}

The above $h_{0}$ represents the constant water depth and the incident wave height $H_{\text {inc }}$ is obtained from the wave height measured by wave gauges $1 . k_{s}$ denotes the effective wavenumber defined in (2.12) and the wave length $L=2 \pi / k_{s}$. For all the cases, the cell porosity is approximately $91.27 \%$.

\subsubsection{Numerical results}

Considering each patch as a homogeneous subzone of the entire forest region, a constant bulk value of dimensional eddy viscosity is assumed and determined by (2.9). Due to the same properties (cylinder arrangement and cell porosity) over four patches, the bulk value of dimensional eddy viscosity is assigned to each patch. Therefore, the cell problem corresponding to each harmonic needs to be solved only once. Note again that the cell problem for different harmonic components $(\omega)$ has to be solved independently because the dimensionless eddy viscosity depends on $\omega$. Following the convergence tests, we here adopt $10^{-3} \leq \omega \leq 15$ (with 40 equally-distributed values) as the arrangement of the truncated harmonic components in numerical computations. For each harmonic, the same discretization of forest boundary (see Chang et al. 2016's Fig.4 or Fig.S4 in the Supplementary Materials) is applied for each patch with 720 boundary elements in total (i.e. $\mathbb{N}_{\xi}=180$ and $\mathbb{N}_{S}=720$ ). Uniform solution along each element is assumed. For each patch (i.e. patch $\xi$ ), the outward normal direction of the boundary can be represented by $r_{\xi}$ which is shown in Fig.4 of Chang et al. (2016) (also shown as Fig.S4 in the Supplementary Materials), i.e. $\hat{n}_{\xi}=-\hat{n}_{\mathrm{S}} \equiv r_{\xi}$. Therefore, the 
matching conditions in (3.15) and (3.16) become

$$
\left(\hat{\mathcal{G}}_{\xi}\right)_{i j}\left(\frac{\partial \hat{\eta}_{\xi}^{(0)}}{\partial r_{\xi}}\right)_{j}=\hat{A}_{0} e^{\mathrm{i} X_{i}}-\left(\hat{\mathcal{Q}}_{\mathrm{S}}\right)_{i m}\left(\frac{\partial \hat{\eta}_{\mathrm{S}}^{(0)}}{\partial r_{\xi}}\right)_{m}
$$

and

$$
-\mathrm{i} \hat{\mathcal{K}}\left(\frac{\partial \hat{\eta}_{\xi}^{(0)}}{\partial r_{\xi}}\right)_{i}=\hat{A}_{0} \frac{\partial e^{\mathrm{i} X_{i}}}{\partial r_{\xi}}+\left(\frac{\partial \hat{\eta}_{\mathrm{S}}^{(0)}}{\partial r_{\xi}}\right)_{i}
$$

where $i, j=1 \cdots \mathbb{N}_{\xi}$ and $m=1 \cdots \mathbb{N}_{S}$ with $\mathbb{N}_{S}=\sum_{1}^{4} \mathbb{N}_{\xi}$. A system of equations for solving $\partial \hat{\eta}_{\xi}^{(0)} / \partial r_{\xi}$ and $\partial \hat{\eta}_{\mathrm{S}}^{(0)} / \partial r_{\xi}$ is formulated. After the unknown $\hat{\eta}_{\xi}^{(0)}$ and $\hat{\eta}_{\mathrm{S}}^{(0)}$ and their normal derivatives along the boundaries are solved, the wave amplitude spectrum in each patch as well as in open water can be obtained from (3.3) and (3.6), respectively. Once again, we note that the wave amplitude spectrum for each harmonic is solved separately and the free surface elevation can be computed by the inverse Fourier transform in (2.14) with numerical integration.

The comparisons of the numerically-simulated free surface elevations with the gauge measurements are presented in Fig.9 to 11. As concluded previously for a circular forest, the nonlinearity of incident wave plays an essential role on the accuracy of model prediction. Similar results can also be observed in Fig.9, 10 and 11. For smaller waves (e.g. Case $1: H_{\text {inc }} / h_{0} \approx 0.15$ ), the largest relative wave height difference appears to be $10 \%$ at wave gauge 7 . For larger wave (e.g. Case $2: H_{\text {inc }} / h_{0} \approx 0.23$ and Case $3: H_{\text {inc }} / h_{0} \approx 0.31$ ), on the other hand, the relative errors increase to $14 \%$ at wave gauge 7 . Furthermore, the discrepancy at wave gauge 4 , which is close to the entry of the vegetated area, is higher than expected while the measurements at wave gauge 10 for Case 2 and 3 present lower values than expectation. It is also obvious that the phase differences between the model predictions and the data become noticeable for all the cases when the wave gauges are outside the vegetated area (i.e. wave gauges $7 \& 24$ ). In fact, the phase differences for three cases are almost the same (i.e. $\approx 0.13 \mathrm{~s}$ at wave gauges $7 \& 24$ ). Finally, we also compare the numerically-simulated wave heights along the centerline with those for a circular forest (Sec.4.2) in Fig.12. Since more cylinders are used for the single circular forest than those for four smaller patches, higher attenuation of the advancing wave crest (around $10 \%$ for Case 1 and $15 \%$ for Case 2) is observed in the single patch case. Also, the phase differences due to the existence of more cylinders (i.e. higher porosity) are recognized. 

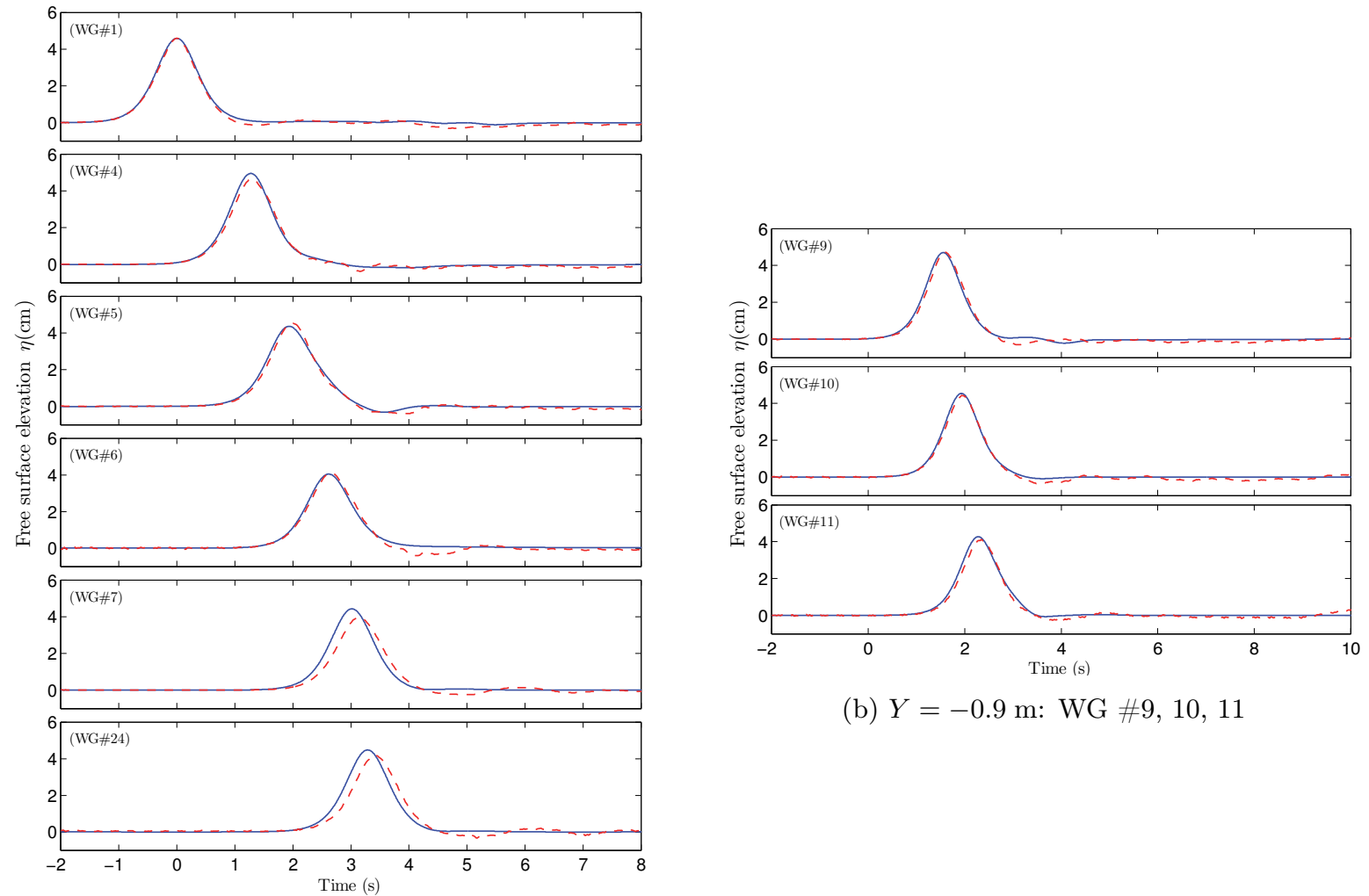

(b) $Y=-0.9 \mathrm{~m}: \mathrm{WG} \# 9,10,11$

(a) $Y=0 \mathrm{~m}$ (Centerline): WG \#1, 4, 5, 6, 7, 24

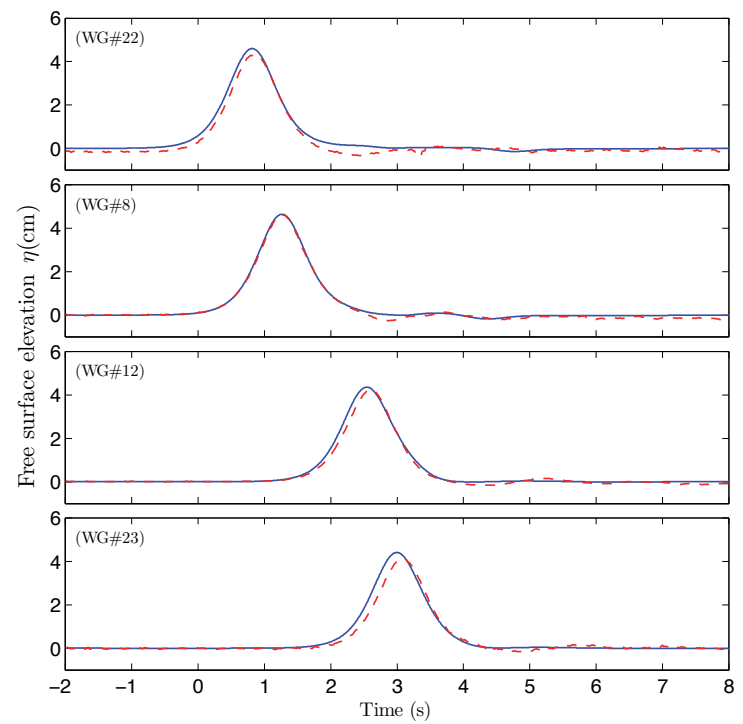

(c) $Y=-1.17 \mathrm{~m}: \mathrm{WG} \# 22,8,12,23$

Figure 9: Comparison of wave height by numerical simulation (solid lines) with experimental data (dashed lines) for multiple patches - Case 1. 

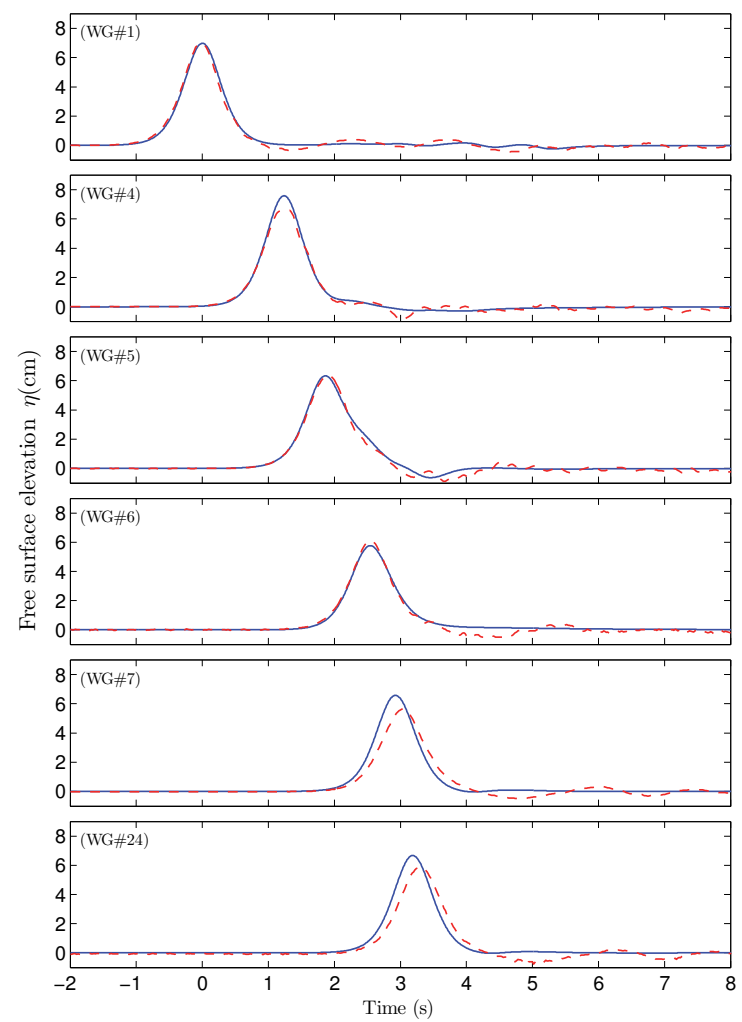

(a) $Y=0 \mathrm{~m}$ (Centerline): WG \#1, 4, 5, 6, 7, 24

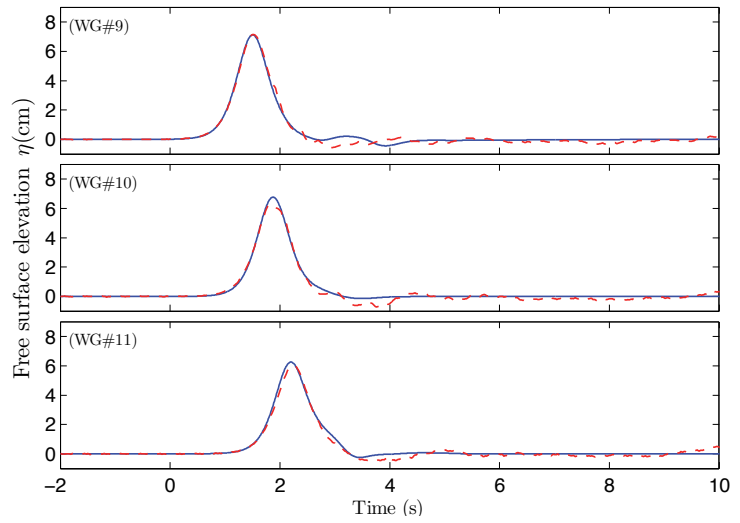

(b) $Y=-0.9 \mathrm{~m}: \mathrm{WG} \# 9,10,11$

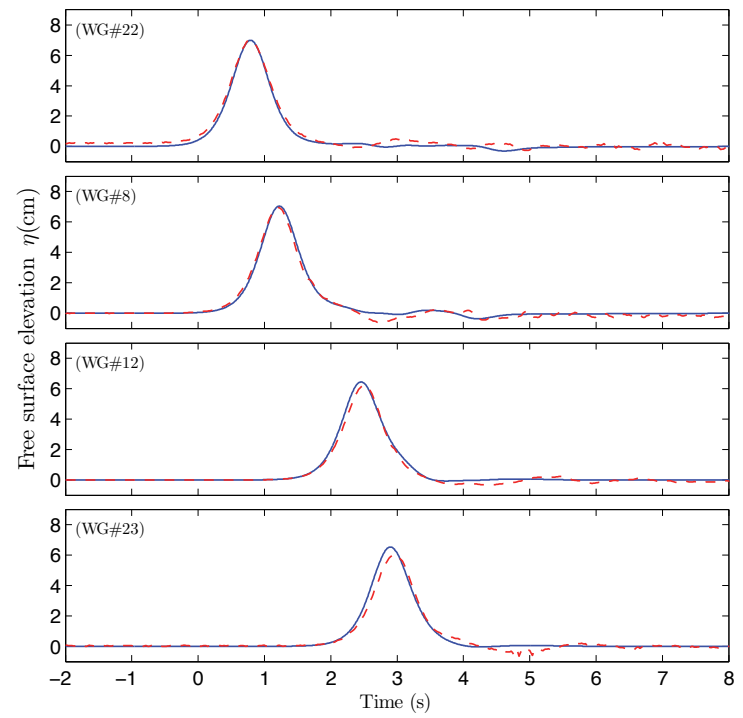

(c) $Y=-1.17 \mathrm{~m}: \mathrm{WG} \# 22,8,12,23$

Figure 10: Comparison of wave height by numerical simulation (solid lines) with experimental data (dashed lines) for multiple patches - Case 2 . 

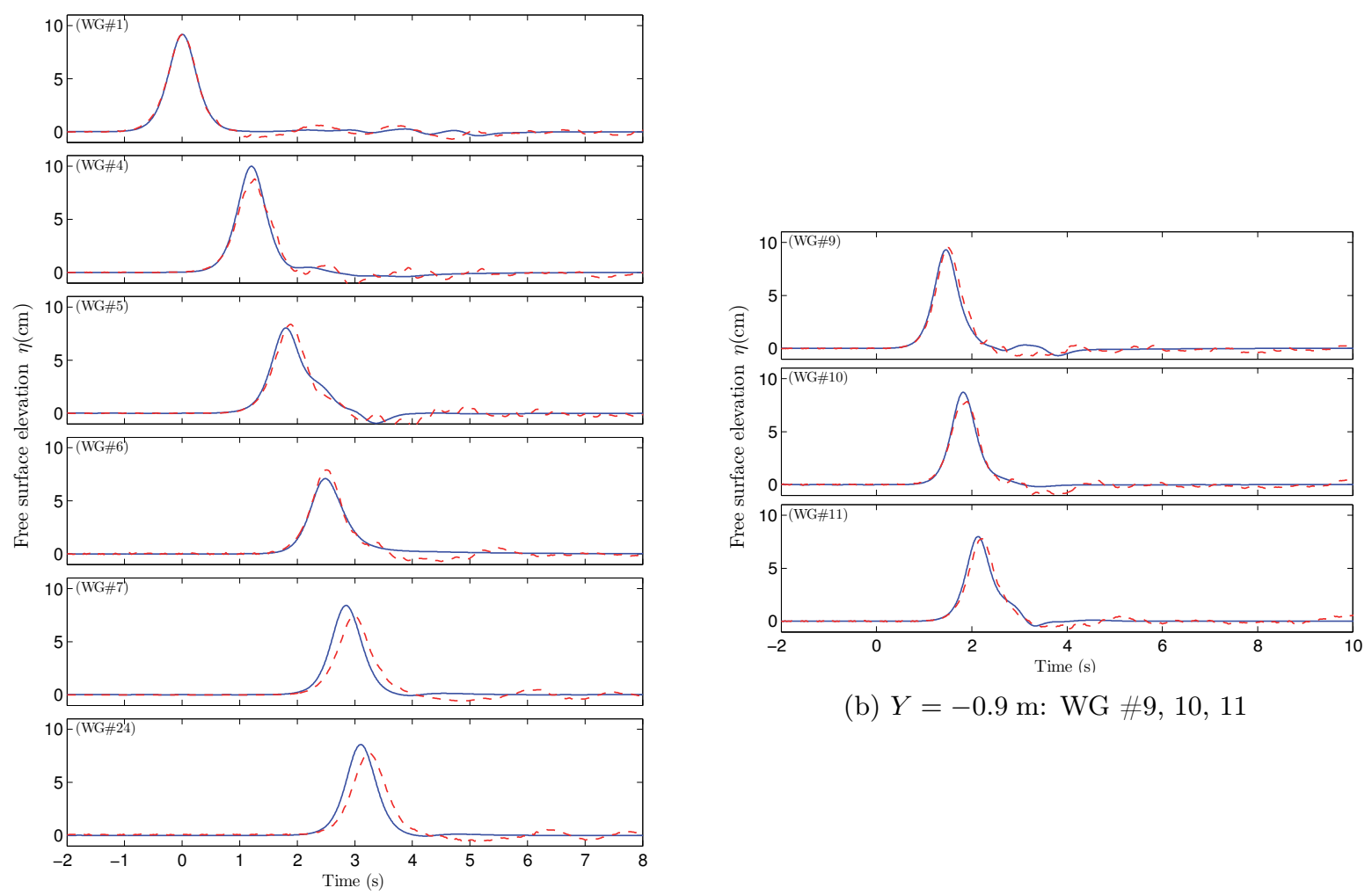

(b) $Y=-0.9 \mathrm{~m}: \mathrm{WG} \# 9,10,11$

(a) $Y=0 \mathrm{~m}$ (Centerline): $\mathrm{WG} \# 1,4,5,6,7,24$

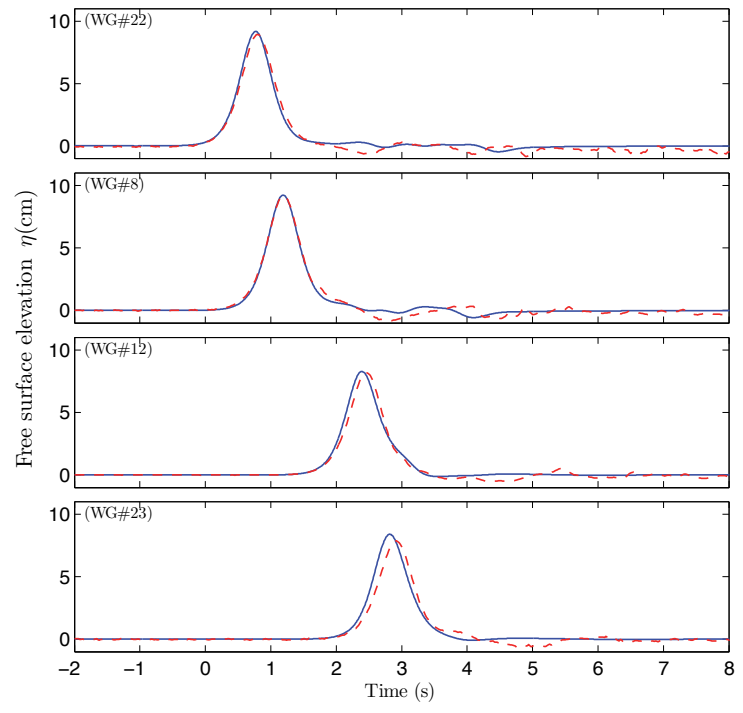

(c) $Y=-1.17 \mathrm{~m}: \mathrm{WG} \# 22,8,12,23$

Figure 11: Comparison of wave height by numerical simulation (solid lines) with experimental data (dashed lines) for multiple patches - Case 3 . 


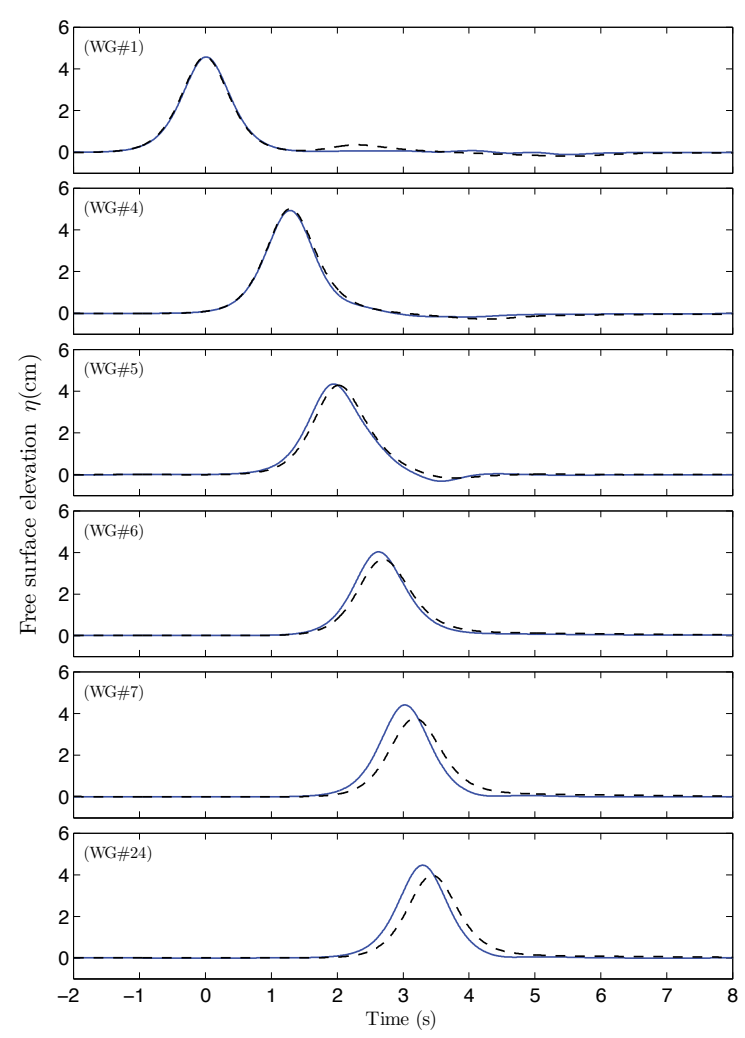

(a) Case 1: $H_{\text {inc }}=4.59 \mathrm{~cm}$

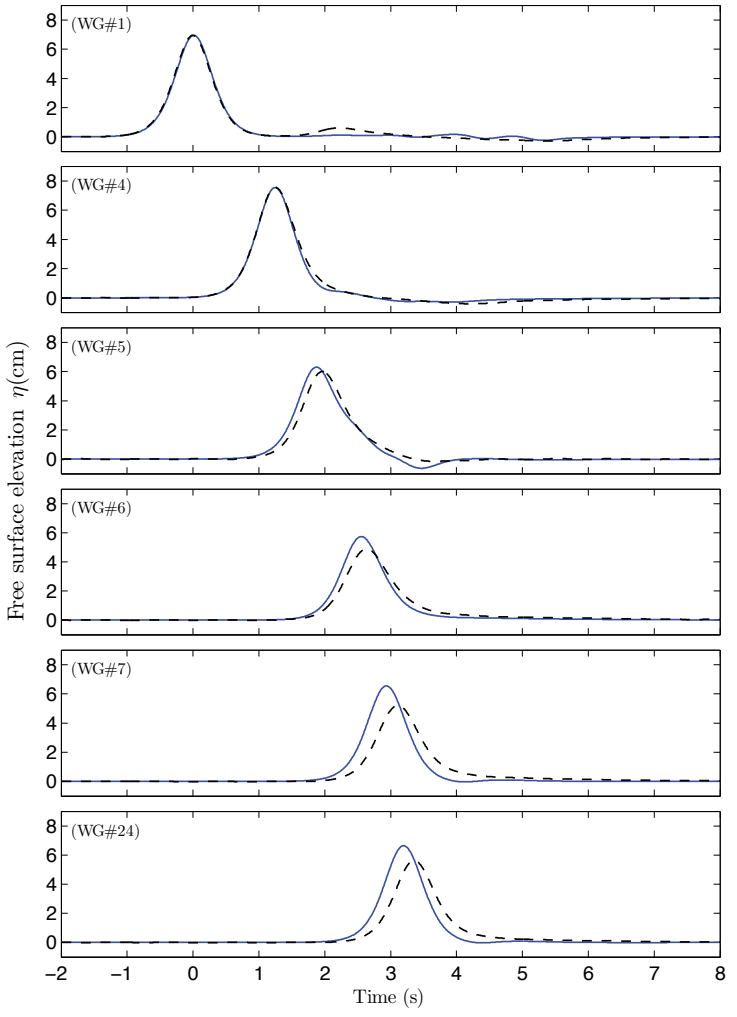

(b) Case 2: $H_{\text {inc }}=6.98 \mathrm{~cm}$

Figure 12: Comparison of numerical-simulated wave heights through the centerline of a circular forest (dashed lines) and of multiple forest patches (solid lines).

\section{Concluding remarks}

The mathematical model developed by Mei et al. (2011) on a transient long wave with a soliton-like impulse propagating through coastal vegetation is extended and further studied in this paper. The multi-scale perturbation method is applied to separate the cylinder and wavelength scales. Starting with linearized equations, Fourier transform is used for solving the transient problem, which is different from the approach used in Mei et al. (2011). The micro- and macro-scale problems for each harmonic are solved independently. Once the wave amplitude spectrum for each harmonic is obtained, the free surface elevation can be computed by inverse Fourier transform. It should be remarked again that the incident wave height has to be reasonably small due to the use of the linear wave theory in this paper. To determine the finite number of harmonic components used in the numerical computations, we first perform a test and conclude that $10^{-3} \leq \omega \leq 10$ (with equally-distributed 40 values in total) is sufficient to reconstruct the incident soliton in (2.11). Using this arrangement in the numerical computations shows convergent simulated free surface elevation only for relatively smaller waves. On the other hand, for larger waves higher values of harmonics (e.g. $10^{-3} \leq \omega \leq 15$ ) are needed such that the range of harmonics has to be modified.

Following Chang et al. (2016), the boundary integral equation method is employed for dealing with the arbitrary forest shape and solving the leading-order wave amplitude spectrum numerically. In this paper, each forest subzone is considered as a homogeneous region where a dimensional bulk eddy viscosity is determined by an empirical formula. It is reiterated here that while the dimensional eddy viscosity is a constant in a subzone, the dimensionless eddy viscosity depends on the harmonic component. Similar to Chang et al. (2016), a computer program has been written based on the present numerical model and can be applied to different configurations of forest patches. The required inputs of the computer program are described in the Appendix. 
The forest belt studied in Mei et al. (2011) is first examined with new analytical solutions. A modification of the empirical formula for eddy viscosity in Mei et al. (2011) shows better agreements between analytical results and reported data. By checking the damping rate, the model limitation on the nonlinearity of the incident wave is observed. We also test the efficiency of different forest sizes on damping the incident wave energy. As expected, thicker forest belts bring about higher wave dissipation. For the largest wave in their cases (i.e. $H_{\text {inc }} / h_{0} \approx 0.1864$ ), approximatedly $90 \%$ of incident wave height is damped out when the forest width becomes four times of the original size (i.e. $4 \times L_{\mathrm{F}}$ ). The circular forest, as presented in Liu et al. (2015) and Chang et al. (2016), is then used to check the numerical model. The numerical results and the experimental data show very good agreement. The relative differences between measured and simulated wave heights are less than $17 \%$. For the cases with smaller nonlinearity, i.e. $H_{\text {inc }} / h_{0} \leq 0.2$, the relative errors become less than $10 \%$. Focusing on the damping rate along the centerline, the relative errors are less than $5 \%$ for most of the cases (i.e. $H_{\text {inc }} / h_{0} \leq 0.3$ ), which further confirms the linear limitation of the present model on the nonlinearity of incident wave. Further investigation on the present model is conducted by using one of Maza et al. (2016)'s experiments, which contains four smaller circular patches. Again, good agreement between model results and the data are found and the effects of the nonlinearity of incident wave are also observed. Similarly, the discrepancy between numerical simulations and the data starts increasing when $H_{\text {inc }} / h_{0}>0.2$. The comparison for wave attenuation by the single circular forest and by multiple forest patches is also presented, showing higher attenuation of incoming waves by the former.

\section{Acknowledgment}

This work has been partially supported by research grants from the National Science Foundation (0925711 and 1041541) to Cornell University. Research funding provided by Atkins Center for Sustainable Future and College of Engineering at Cornell University for performing experiments at University of Cantabria is also acknowledged. CCM was also funded by a Mary Upson Visiting Professorship from Cornell University.

\section{A Inputs/outputs for the numerical model}

The inputs for running the present model have been given in Chang et al. (2016), including the incident wave condition, forest configuration and the computational domain. All the input files have the same contents and formats except the incident wave condition file WaveInc.dat, which has to be modified as $\rightarrow\left\{h_{0} ; H_{\text {inc }} ; \mathrm{N}_{\omega}\right\}$ with $\mathrm{N}_{\omega}$ denoting the total number of harmonics being used in the computation. In addition, one more input file including an array of the harmonic components is required: Omega.dat $-\{\omega\}$. More details can be referred to the Supplementary Materials. On the other hand, the output of the model has only one file - etaG.dat, which is a three-dimensional array containing the leading-order dimensionless free surface elevation in the entire computational domain at different time. It has size $\mathrm{N}_{\mathrm{Yc}_{\mathrm{c}}} \times \mathrm{N}_{\mathrm{Xc}} \times \mathrm{N}_{\mathrm{T}}$ where $\mathrm{N}_{\mathrm{T}}$ denotes the number of grids in time. The cell problem solver is an open source finite element software - FreeFEM ++ (Hecht 2012). Several different cell configurations have been tested.

\section{References}

Chang, C.-W., Liu, P. L.-F., Mei, C. C., and Maza, M. Periodic water waves through a heterogeneous coastal forest of arbitrary shape. Coastal Engineering, 2016. Submitted.

Hecht, F. New development in freefem++. Journal of Numerical Mathematics, 20:251-265, 2012.

Huang, Z., Yao, Y., Sim, S. Y., and Yao, Y. Interaction of solitary waves with emergent, rigid vegetation. Ocean Engineering, 38:1080-1088, 2011.

Husrin, S., Strusinska, A., and Oumeraci, H. Experimental study on tsunami attenuation by mangrove forest. Earth, Planets and Space, 64:973-989, 2012.

Irtem, E., Gedik, N., Kabdasli, M. S., and Yasa, N. E. Coastal forest effects on tsunami run-up heights. Ocean Engineering, 36:313-320, 2009. 
Ismail, H., Wahab, A. K. A., and Alias, N. E. Determination of mangrove forest performance in reducing tsunami run-up using physical models. Natural Hazards, 63:939-963, 2012.

Liggett, J. A. and Liu, P. L.-F. The boundary integral equation method for porous media flow. Allen \& Unwin, 1983.

Liu, P. L.-F., Chang, C.-W., Mei, C. C., Lomonaco, P., Martin, F. L., and Maza, M. Periodic water waves through an aquatic forest. Coastal Engineering, 96:100-117, 2015.

Maza, M. Experimental and numerical modelling of flow interaction with natural ecosystems for coastal protection. PhD thesis, University of Cantabria, 2015.

Maza, M., Lara, J. L., and Losada, I. J. Tsunami wave interaction with mangrove forests: A 3-d numerical approach. Coastal Engineering, 98:33-54, 2015.

Maza, M., Lara, J. L., and Losada, I. J. Solitary wave attenuation by vegetation patches. Advances in Water Resources, 98:159-172, 2016.

Mei, C. C. and Vernescu, B. Homogenization methods for multiscale machanics. World Scientific, 2010.

Mei, C. C., Chan, I.-C., Liu, P. L.-F., Huang, Z., and Zhang, W. Long waves through emergent coastal vegetation. Journal of Fluid Mechanics, 687:461-491, 2011.

Mei, C. C., Chan, I.-C., and Liu, P. L.-F. Waves of intermediate length through an array of vertical cylinders. Environmental Fluid Mechanics, 14:235-261, 2014.

Miles, J. W. Damping of weakly nonlinear shallow-water waves. Journal of Fluid Mechanics, 76:251-257, 1976.

Strusinska-Correia, A., Husrin, S., and Oumeraci, H. Tsunami damping by mangrove forest: a laboratory study using parameterized trees. Natural Hazards, 13:483-503, 2013. 